Article

\title{
Evaluation of the Efficacy of SIFT-MS for Speciation of Wastewater Treatment Plant Odors in Parallel with Human Sensory Analysis
}

\author{
Vaughan S. Langford ${ }^{1, * \mathbb{D}}$, Cassandra Billiau ${ }^{2}$ and Murray J. McEwan ${ }^{1,3}$ \\ 1 Syft Technologies Limited, 3 Craft Place, Middleton, Christchurch 8024, New Zealand; \\ murray.mcewan@canterbury.ac.nz \\ 2 Watercare Services Limited, 73 Remuera Road, Remuera, Auckland 1050, New Zealand; \\ Cassandra.Billiau@water.co.nz \\ 3 Department of Chemistry, University of Canterbury, Christchurch 8000, New Zealand \\ * Correspondence: vaughan.langford@syft.com; Tel.: +64-3-338-6701
}

Received: 16 September 2020; Accepted: 12 October 2020; Published: 15 October 2020

\begin{abstract}
Odors from wastewater treatment plants (WWTPs) have frequently been attributed primarily to hydrogen sulfide $\left(\mathrm{H}_{2} \mathrm{~S}\right)$. Low-to-medium cost hydrogen sulfide sensors have been utilized as odor indicators. However, other odorous species are usually present that may have lower odor thresholds than hydrogen sulfide. Hydrogen sulfide is not always present in odorous environments and the correlation of hydrogen sulfide to odor at a treatment facility is inconsistent. Such factors determine hydrogen sulfide an inconsistent indicator and more sophisticated measurement techniques are required to accurately predict odor intensity from complex gaseous mixes. In this paper, the performance of a direct mass spectrometric technique, selected ion flow tube mass spectrometry (SIFT-MS), is evaluated for analysis of odors from diverse sources at a modern WWTP. The soft chemical ionization employed in SIFT-MS provides detection and quantification of a wide range of potential odorants to below, or close to, the human odor detection threshold (ODT). The results presented demonstrate that methyl mercaptan is almost always a more significant odorant at this WWTP than hydrogen sulfide and confirm that the relative abundances of these odorants vary significantly. Parallel SIFT-MS chemical analysis and human sensory analysis (olfactometry) was conducted in this study. Good agreement was observed for samples of moderate to strong "sewage" or "chemical" character. However, in samples that were otherwise low in odor intensity, sensory analysis did not attribute "sewage" odor notes as the predominant odor character. Chemicals attributed with this odor character were present significantly above the ODTs in the mixed samples and were detected by SIFT-MS. A weak correlation was obtained between total odor activity values measured using SIFT-MS and the odor concentration (in odor units per cubic meter) determined using dilution olfactometry. The complexity of the wastewater matrix and complexity of human odor recognition from mixed samples is thought to be the underlying cause of less-than-ideal correlation, perturbing both olfactometry and SIFT-MS analyses.
\end{abstract}

Keywords: SIFT-MS; sensory analysis; wastewater treatment; odor; volatile organic compounds; volatile sulfur compounds

\section{Introduction}

Wastewater entering most treatment plants is predominantly a varying mixture of both industrial wastewater (so-called trade wastes), residential (domestic) wastes, and runoff from rainfall. As such, it comprises a diverse and varying matrix for a wastewater treatment plant (WWTP) to process [1]. Due to the organic content, chemical content, and high microbial load in the influent and through much 
of the wastewater treatment process, both odor monitoring and mitigation is important. Odor regulation ensures WWTPs are not an odor nuisance to nearby business and residential areas. Mitigation strategies include containing odorous air using sealed lids, enclosing in an air-tight structure and ducting the air through biofilters in which microbes metabolize the odorants.

Modern WWTPs involve multistep waste processing [1,2]. First, the influent is screened for objects greater than a few millimeters diameter; these screenings and grit are collected and then sent to landfill. The screened influent undergoes primary treatment through sedimentation. Solids are passed to secondary solids treatment, while the supernatant liquid is pumped to reactor-clarifiers. Solids undergo various thickening processes (e.g., through gravity thickener belts and centrifugation), stabilization (e.g., with lime), and finally re-use or disposal. Meanwhile, secondary water treatment process involves wastewater passing through multiple aerobic and anaerobic cycles containing activated sludge prior to clarification, disinfection and discharge. Due to each process releasing various mixtures of odorants as different components break down at various stages, concentrations of odorous compounds and their observed overall offensiveness can differ significantly. Further impeding prediction of odor are factors such as influent composition and weather conditions. Complicating generalization across plants are different processing approaches and climate effects. Odorous gas emitted at each stage is commonly passed through biofilters or bio-scrubbers. Therefore, odor emission rates at each point are not indicative of raw odor generated at this stage of the process but are an indication of treated odorous gas.

Despite the process complexities, hydrogen sulfide has consistently been used in the industry as an indicator of odor [1]. Low-to-medium cost sensors have been utilized widely to monitor hydrogen sulfide for this purpose [3]. However, other odorous species are also usually present and can have lower odor thresholds than hydrogen sulfide [4]; for example, methyl mercaptan. The biosolids containment shed has consistently displayed high odors when measured with olfactometry, while low levels of hydrogen sulfide have been detected from ambient air at this site on numerous occasions $(<10 \mathrm{ppbV})$ (operational data from internal study at plant, current SIFT-MS data from this study). Hydrogen sulfide as an indicator has its challenges as it is not always present in odorous environments and the correlation of hydrogen sulfide with odor is inconsistent $[5,6]$. More sophisticated measurement techniques are required to better represent complex odor from complex wastewater processes.

Odor analysis using sensor technologies has proved challenging. ENoses (or technical sensors) received significant attention in the late 1990s and 2000s [1] as a solution to modelling and predicting complex odor. These devices still do not qualify as equivalent to human noses for olfactometry measurements [7]. Conventional laboratory analysis of odors using chromatographic methods can be expensive due to the need for multiple analyses for the chemically diverse odor species (ranging from reduced sulfur compounds, to amines, to aldehydes, to volatile fatty acids). Furthermore, laboratory analysis is limited to grab samples due to this complexity and the nature of the conventional chromatographic instrumentation. Hence, modelling complex changes in odor over time is extremely challenging.

Since the late 2000s, direct mass spectrometry (DMS) techniques have become more mainstream in environmental monitoring [8] and identification of odors in food sciences [9]. DMS methods can analyze odor in real-time and can be deployed at site. Proton transfer reaction mass spectrometry (PTR-MS) has been the most widely applied DMS technique for environmental monitoring [10-14]. PTR-MS has specifically been used to optimize effluent control biofiltration systems at an intensive pig production facility using static and mobile laboratory methods [15,16].

Selected ion flow tube mass spectrometry (SIFT-MS) was commercialized by Syft Technologies more recently than PTR-MS. SIFT-MS (Figure 1) has a potential advantage over PTR-MS due to its multiple, rapidly switchable chemical ionization agents (so called reagent or precursor ions) that detect diverse odor compounds with high selectivity [15,17-19]. The SIFT-MS technique has been described in detail elsewhere [20,21]. Briefly, the SIFT-MS reagent ions are generated by a microwave plasma through moist air, then selected individually using a quadrupole mass filter. 
The mass-selected reagent ions first encounter carrier gas, which collisionally cools the ions and contributes to consistent ionization and quantitation from reagent ion-analyte reactions occurring when the sample is introduced. After a few milliseconds of reaction, remaining reagent ions and product ions are mass-filtered and detected, with concentrations calculated point-by-point. Data obtained by SIFT-MS instruments are congruent with an accepted chromatographic method for environmental volatile organic compound (VOC) analysis [22].

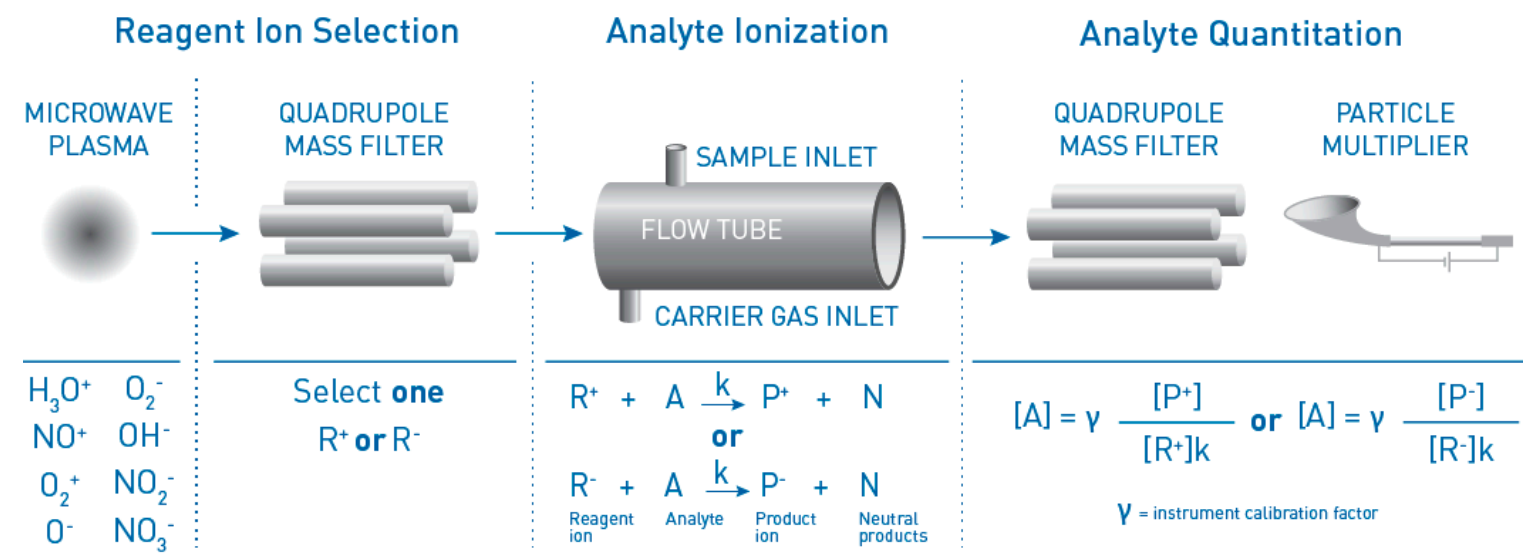

Figure 1. Schematic representation of the SIFT-MS analytical technique, which is based on direct, soft sample ionization with atomic and molecular ions. Used with permission of Syft Technologies Limited.

Braithwaite's review of the odor analysis literature [5] has noted the potential of SIFT-MS for odor analysis in parallel with gas chromatography-mass spectrometry/olfactometry (GC-MS/O) and sensory analysis (the odor profile method (OPM) in particular). GC-MS/O separates volatile compounds via gas chromatography, determines their mass by mass spectrometry and evaluates their odor strength and characterization via sensory evaluation (olfactometry) $[23,24]$. In addition to the complexities of chemical odorant species detection, the chemical, physiological and neurological mechanisms involved in odor perception in humans are complex and yet to be fully understood. Of the $>1000$ known genes encoding olfactory receptors in the human genome (the largest multigene family), most are found in gene clusters and only 388 of these may be functional [25]. This may be due to coding region disruptions that may create non-functional pseudogenes [25,26]. A comparison of 20 receptor genes from human to chimpanzees showed the loss of function on half of the chosen receptors in humans when compared to the functioning gene in chimpanzees [26]. Genetic variation between family members has been studied and a hereditary link was found between the linkage of pleasantness to cinnamon odors and the perceived intensity of chocolate, rose and paint thinner [27]. Therefore, it is probable that genetic variation within humans plays a role in odor assessment and perceived intensity of individual odors.

When attempting to generate algorithms that replicate odor receptor responses, there is no linear correlation between sensory input and odor mapping in the brain. For example, a single odor receptor such as hOR1G1 can recognize a large spectrum of odors that stimulate many different olfactophores in the olfactory bulb [28]. This receptor was also suggested to have differing affinity to different odorants at its receptor binding site inferring that in a complex mix, some odorants would have preferential binding due to their affinity to the binding site. It was also suggested that binding was easily reversible due to the hydrogen binding associated with the binding site [28]. Much work is needed before the physiological workings of each receptor expressed at any one time in any of the millions of olfactory sensory neurons present in human noses is fully understood. In addition, each olfactory sensory neuron has a three-month life span and is regenerated over the human lifetime. A negative feedback loop is used to ensure only one type of olfactory receptor is ever expressed in a cell at one time [29]. Hence, with this complexity and variation between individuals, it is difficult to generate standard algorithms to predict odor responses of much of the human population. 
This paper seeks to bridge the gap between sensory and instrumental methods in a way that has not been previously possible because of the limitations of conventional instrumental techniques. It first describes the first application of SIFT-MS to identify probable odor-causing chemical species at a modern WWTP, evaluating its performance against the extant literature for chemical odorant analysis in WWTPs. Then a comparison is made of SIFT-MS against odor concentration from dynamic dilution olfactometry, and against informal odor notes. These evaluations were conducted alongside an existing biennial odor emissions survey at the WWTP. The results presented here indicate the correlation of chemical composition provided by SIFT-MS analysis to final odor emissions (post odor treatment) at different stages of the plant. However, this four-month study indicated that SIFT-MS could be developed into a useful tool to assist in making operational decisions regarding odor mitigation strategies and assist with planning for future odor mitigation infrastructure (i.e., developing most suitable bio-scrubber media suited to the site). SIFT-MS analysis may be used for continuous monitoring on site to assess the effect of operational changes on odor emissions from isolated parts of the plant. SIFT-MS analysis may also be used to monitor biogas composition and determine the efficiency of the siloxane scrubber that reduces organosilicon-based engine wear. SIFT-MS data may also assist with furthering the capability of wastewater treatment process modelling software as it can provide additional indicators of the changes to dominant biochemical pathways involved in processes. Further validation and modelling would be required before the technique could provide online feedback and alerts to assist with operational process optimization.

\section{Materials and Methods}

\subsection{Wastewater Treatment Plant and Sampling Design}

The modern New Zealand WWTP investigated in this study treats wastewater from both industrial and residential sources. The odor survey was conducted over the Southern Hemisphere summer and early autumn/fall period (December 2018-April 2019) and focused on odor emission sources post-treatment at the plant using olfactometry analysis. Parallel samples were collected for SIFT-MS chemical analysis in the same material (nalophan bag). Samples for comparative analysis were obtained from a wide range of potential odor sources at the plant including odor beds and reactor clarifiers. Figure 2 shows a schematic diagram of the WWTP process. Raw sewage (influent) was screened for debris and grit, then processed via primary sedimentation. The liquid was pumped for treatment in the secondary liquids activated sludge process, where the process included repeated aerobic and anaerobic cycling prior to clarification and sterilization. The solids were passed to the secondary solids process, in which thickening, anaerobic digestion, dewatering and stabilization steps were undertaken prior to disposal of the biosolids. The biogas generated during anaerobic digestion was combusted for energy generation.

Tables 1-3 summarize the process locations corresponding to sampling using flux chambers on biofilters, flotation chambers on liquid surfaces, and stack/ambient gases, respectively. These tables also introduce a four-letter code that is used to identify locations in abbreviated form in subsequent text, tables, and figures (the "Location ID"; e.g., IP-EF for the earth filters on the screens in the influent/primary treatment phase). 
Table 1. Process locations for biofilters that were sampled in this study (using the flux chamber approach according to AS/NZS 4323.4:2009) and biofilter compositions.

\begin{tabular}{ccccc}
\hline Treatment Phase & Location(s) & $\begin{array}{c}\text { Location } \\
\text { ID }\end{array}$ & $\begin{array}{c}\text { Number of } \\
\text { Samples }\end{array}$ & $\begin{array}{c}\text { Biofilter } \\
\text { Type }\end{array}$ \\
\hline Influent and Primary & Screens, Primary tanks & IP-EF & 13 & A \\
Primary & Pumping station & P-PS & 2 & A \\
Primary & Splitter box for Reactor-Clarifiers & P-SP & 6 & A, B, C \\
Secondary solids & Gravity thickeners & SS-GT & 5 & D \\
Secondary solids & Digester Feed & SS-DF & 3 & A \\
Secondary solids & Centrifuge & SS-CE & 3 & A \\
Secondary solids & Biosolids storage & SS-BS & 10 & D \\
Secondary solids & Ammonia scrubber & SS-AS & 4 & \\
\hline Total samples & & 46 & \\
\hline 1 Biofilter types: A $=70 \%$ Scoria (AP7), 30\% Aged Bark; B = 37\% Coarse Scoria (50/20 mm), 37\% Fine Scoria \\
(20/7 mm), 26\% Compost/Pumice Mix (50-50 Standard Compost \& 1-3 mm Pumice; C = Bark, Compost/Pumice mix; \\
D = 37\% Coarse Scoria (50/20 mm), 37\% Fine Scoria (20/7 mm), 26\% Compost/Pumice Mix (50-50 Standard Compost \\
$\& 1-3$ mm Pumice).
\end{tabular}

Table 2. Process locations for flotation samples that were collected during this study (using the flux chamber approach according to AS/NZS 4323.4:2009 [30]).

\begin{tabular}{cccc}
\hline Treatment Phase & Location(s) & Location ID & Number of Samples \\
\hline Secondary water & Reactor aerobic location 1 & SW-A1 & 8 \\
Secondary water & Reactor aerobic location 2 & SW-A2 & 9 \\
Secondary water & Reactor anoxic location 1 & SW-AO & 9 \\
Secondary water & Clarifier & SW-CL & 20 \\
Secondary solids & Dissolved air flotation & SS-AF & 6 \\
\hline Total samples & & & 52 \\
\hline
\end{tabular}

Table 3. Process locations for stack and ambient samples collected during this study (according to AS/NZS 4323.3:2001 [31]).

\begin{tabular}{|c|c|c|c|c|}
\hline Treatment Phase & Location(s) & Location ID & $\begin{array}{l}\text { Number of } \\
\text { Samples }\end{array}$ & Sample Type \\
\hline Influent-Primary & Screens building & IP-SB & 2 & Ambient bag \\
\hline Secondary solids & $\begin{array}{l}\text { Gas turbine stack, } \\
\text { post biogas combustion }\end{array}$ & SS-TS & 3 & Stack bag via port \\
\hline Secondary solids & $\begin{array}{c}\text { Sludge stack, } \\
\text { biosolids building }\end{array}$ & SS-SS & 4 & Stack bag via port \\
\hline Total samples & & & 9 & \\
\hline
\end{tabular}

\subsection{Sampling Procedures}

Sampling was carried out on liquid surfaces (i.e., dissolved air flotation (DAF) tanks in secondary solids treatment and reactor clarifier tanks in secondary water treatment) using a United States Environmental Protection Agency (US EPA) static flux chamber (SFC) to undertake the monitoring [32]. For sources with positive gas release (i.e., the biofilter outlets) an open hood sampling method was used. In both cases, sampling was in conformance with procedures described in AS/NZS 4323.4:2009 [30].

Engine stack and sludge handling stack samples were collected using a point source emission sampling method as described in AS/NZS 4323.3:2009-Stationary Source Emissions Part 3: Determination of Odor Concentration by Dynamic Olfactometry [31]. 


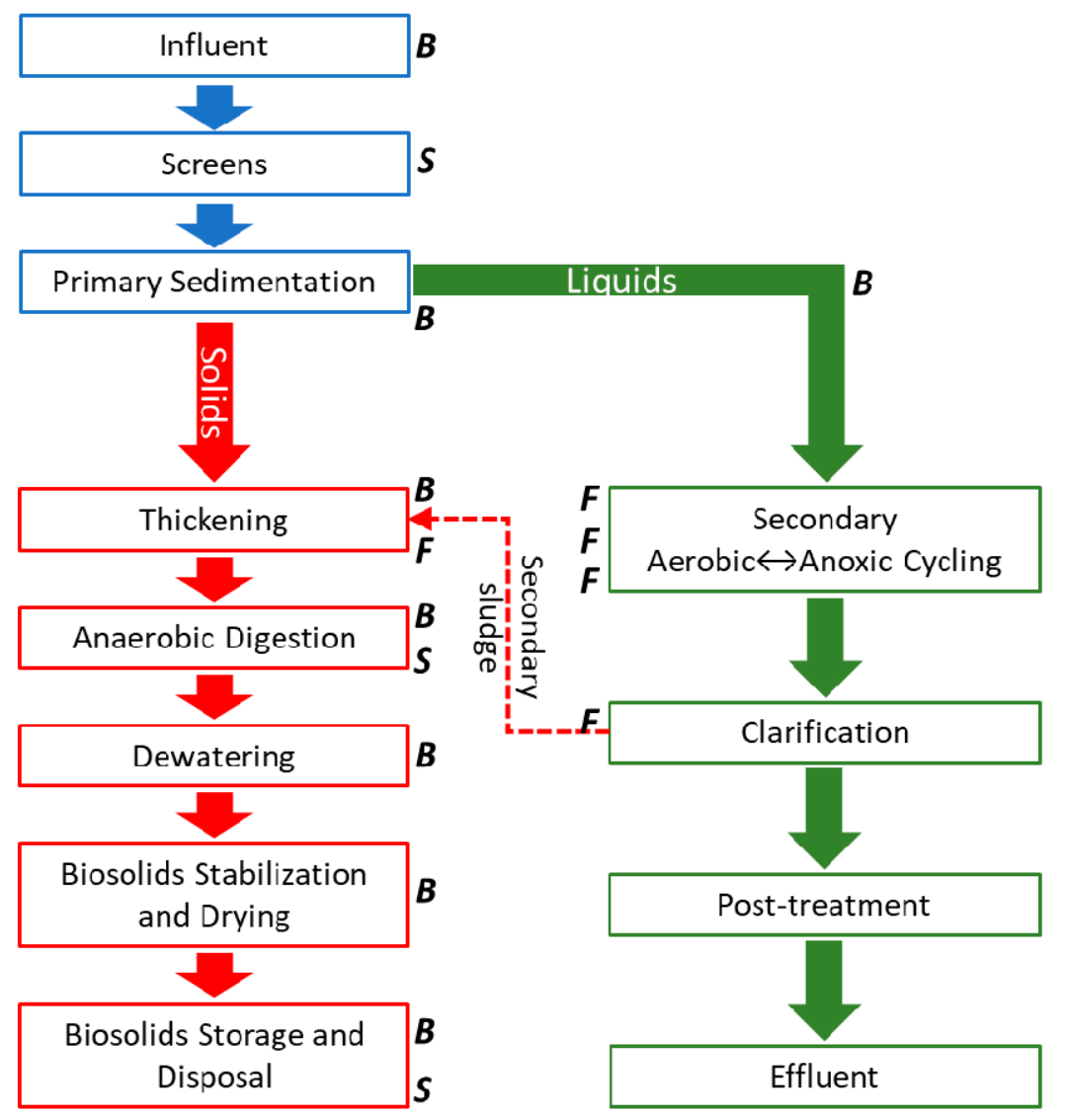

Figure 2. Schematic diagram of the process at the WWTP investigated in this study. Sampling method

(Tables 2-4) is indicated as B = biofilter, $\mathrm{F}=$ flotation and $\mathrm{S}=$ stack/ambient.

In all cases, Nalophan NA odor bags (made in house from film supplied by Coimex International Limited, Brazil) were used to contain air samples for both olfactometry and instrumental analysis. Sampling lines were made from PTFE tubing and used once. Each collection apparatus was placed and left to condition prior to collecting samples. Collection of samples was via a lung system where a vacuum was applied to the sample barrel resulting in the bag 'lung' filling with sample air without contamination from the pump. Bags were filled with sample, conditioned and emptied. The conditioned bags were then filled with sample for analysis using gas-sampling pumps with calibrated flows [30].

Samples for both sensory and instrumental analysis were analyzed within $24 \mathrm{~h}$ of sampling.

\subsection{Sensory Analysis}

Olfactometry was conducted at the Watercare Laboratory Services Odor Laboratory. Odor concentrations (in odor units $\mathrm{m}^{-3} ; \mathrm{OU} \mathrm{m}^{-3}$ ) were determined using dilution olfactometry according to AS/NZS 4323.3:2001 [31]. An Olfasense olfactometer (Kiel, Germany, www.to-evolution.com) was used with four to six panelists.

Assessment of odor character was conducted informally according to an in-house laboratory procedure. The odor characteristic is a qualitative measurement of what is predominantly perceived by the odor panelists. After dilution olfactometry is completed, the sample is presented to all odor panelists to carefully sniff the undiluted sample. The panel must reach a consensus on the predominant odor character and its intensity. An odor character description is provided to the technician for each sample and the panel are not informed of the source of the odor. 


\subsection{SIFT-MS}

SIFT-MS data for this article were obtained using a Voice200ultra SIFT-MS instrument (Syft Technologies, Christchurch, New Zealand; www.syft.com) in positive ion mode. Target compounds for SIFT-MS analysis were derived from References [1], [2] and [4]. The reagent-product ion pairs for SIFT-MS detection of each target compound are summarized in Table 4. Other SIFT-MS product ions were usually available but as they were not utilized in determining the final concentrations, they have been omitted for clarity.

Quantitation for most compounds was carried out from the SIFT-MS library [22]. Hydrogen sulfide, methyl mercaptan and ethyl mercaptan + dimethylsulfide, and dimethyl disulfide were calibrated against certified gas mixtures (CAC Gas, Sydney, Australia) to improve accuracy. Limits of quantitation (LOQs) were estimated as one-half the mean blank concentration. Due to sampling from bags, minimum values for LOQs were set at 0.2 parts-per-billion by volume ( $\mathrm{ppbV}$ ) for targeted compounds (selected ion mode, SIM) and $0.5 \mathrm{ppbV}$ for compounds processed subsequently from full scan data. LOQs are shown in Table 4. Blank bags were filled with odor-free scrubbed air used in olfactometry and provided a baseline of contamination from the sampling method.

To facilitate comparison of the SIFT-MS data with the sensory results, concentrations were converted to odor activity values (OAVs), by dividing the concentration measurement by the odor detection threshold (ODT). Table 4 summarizes ODT concentrations [33] and odor notes for target compounds, where these are known. Wherever possible ODTs were derived from Nagata [34]. However, where data from Nagata [34] were missing, or were at significant variance compared with other post-1970 values, an alternative recent source was utilized. Where isomers or isobaric compounds cannot be resolved with SIFT-MS and are expected in samples, values for all were compared and a representative mid-range value was utilized (e.g., for the monoterpenes, $\beta$-pinene). However, it should be noted that for many compounds their published ODTs do vary considerably, so OAVs are viewed as indicative. 
Table 4. Target compounds for SIFT-MS analysis, together with published odor detection thresholds (ODTs) and odor notes. Reagent ions and primary product ions mass-to charge ratios (in Daltons) used to quantify target compounds, with secondary (water cluster) ions shown in parentheses. To avoid unnecessary complications, not all ion products of each volatile are shown. More details are given in the text.

\begin{tabular}{|c|c|c|c|c|c|c|c|c|c|}
\hline \multirow[t]{2}{*}{ Compound } & \multirow[b]{2}{*}{$\begin{array}{c}\text { ODT/ppbV @ } \\
25{ }^{\circ} \mathrm{C}\end{array}$} & \multicolumn{3}{|c|}{ Sensory } & \multicolumn{4}{|c|}{ SIFT-MS $^{1}$} & \multirow[b]{2}{*}{ LOQ/ppbV } \\
\hline & & Ref. ${ }^{2}$ & Odor Note & Ref. $^{3}$ & $\mathrm{H}_{3} \mathrm{O}^{+}$ & $\mathrm{NO}^{+}$ & $\mathrm{O}_{2}{ }^{+}$ & Ref. ${ }^{4}$ & \\
\hline \multicolumn{10}{|l|}{ Sulfur-containing } \\
\hline Hydrogen sulfide & 0.41 & 1 & Rotten eggs & I & 35 & & & A & 0.3 \\
\hline Methyl mercaptan & 0.071 & 1 & Decayed cabbage, rotten garlic, sulfur, sweaty & I, II & 49 & & 48 & A & 0.3 \\
\hline $\begin{array}{l}\text { Dimethyl sulfide + } \\
\text { ethyl mercaptan }\end{array}$ & 3.0 & 1 & Decayed vegetables/cabbage, seaweed, garlic & I, II & 63 & 62 & 62 & B & 0.5 \\
\hline Dimethyl disulfide & 2.2 & 1 & Putrification, rotting seaweed, egg & I, II & 95 & 94 & 94 & $\mathrm{~B}$ & 0.2 \\
\hline Dimethyl trisulfide & 1.5 & 1 & Rotten cabbage / vegetables, onions & II & 127 & 126 & 126 & $\mathrm{C}$ & 0.5 \\
\hline Allyl methyl sulfide & 6.1 & 2 & Bad breath, acrid sweat & II & 89 & & 88 & $\mathrm{D}$ & 1.6 \\
\hline Diethyl disulfide & 0.024 & 1 & Gassy, ripe onion, greasy, garlic & III & 123 & 122 & & $\mathrm{E}$ & 0.5 \\
\hline Carbon disulfide & 210 & 1 & Sweet, decayed vegetables & II & & & 76 & $\mathrm{E}$ & 7.9 \\
\hline Propyl mercaptans & 0.51 & 3 & Unpleasant & $\mathrm{I}$ & & 76 & & $\mathrm{~A}, \mathrm{E}$ & 0.5 \\
\hline Butyl mercaptans & 0.81 & 4 & Unpleasant & I & 91 & 90 & & E & 0.4 \\
\hline \multicolumn{10}{|l|}{ Nitrogen-containing } \\
\hline Ammonia & 1600 & 1 & Sharp, pungent & I & $18(36)$ & & $17(18,35,36)$ & $\mathrm{F}$ & 10.1 \\
\hline Methylamine & 36 & 1 & Fishy & I & & 31 & & G & 1.6 \\
\hline Dimethylamine & 32 & 1 & Fishy & I & 46 & & & $\mathrm{~F}$ & 11.2 \\
\hline Trimethylamine & 2.4 & 5 & Fishy, ammoniacal & I & 60 & 59 & & $\mathrm{~F}$ & 2.3 \\
\hline Diethylamine & 47 & 1 & Fishy, ammoniacal & III & 74 & & & $\mathrm{~F}$ & 7.6 \\
\hline Triethylamine & 5.3 & 1 & Fishy, ammoniacal & III & 102 & 101 & & $\mathrm{~F}$ & 0.2 \\
\hline Isopropylamine & 25 & 1 & Fishy, ammoniacal & III & & 44 & & $\mathrm{~F}$ & 3.5 \\
\hline Putrescine & 2.8 & 6 & Decomposing meat & $\mathrm{I}$ & 71 & 69,99 & & $\mathrm{D}$ & 2.0 \\
\hline Cadaverine & 2.4 & 7 & Decomposing meat & I & & 87 & 88 & $\mathrm{D}$ & 1.3 \\
\hline Indole & 0.29 & 1 & Faecal, nauseating & I & 118 & 117 & 117 & $\mathrm{H}$ & 0.2 \\
\hline Skatole & 0.093 & 8 & Faecal, nauseating & I & 132 & 131 & 131 & $\mathrm{H}$ & 0.2 \\
\hline Pyridine & 62 & 1 & Disagreeable, irritating & I & 80 & 109 & 79 & $\mathrm{~F}$ & 0.2 \\
\hline \multicolumn{10}{|l|}{ Volatile fatty acids } \\
\hline Acetic acid & 6.1 & 1 & Vinegar, acrid, plastic & $\mathrm{I}, \mathrm{II}$ & $61(79)$ & 90 & & $\mathrm{I}$ & 1.9 \\
\hline Butyric acid & 0.19 & 1 & Rancid, sweaty, chemical, green & I, II & 89 (107) & 118 & & $\mathrm{I}, \mathrm{J}$ & 0.6 \\
\hline Valeric acid & 0.038 & 1 & Sweaty & I & 103 & & & $\mathrm{E}$ & 0.8 \\
\hline
\end{tabular}


Table 4. Cont.

\begin{tabular}{|c|c|c|c|c|c|c|c|c|c|}
\hline \multirow[t]{2}{*}{ Compound } & \multirow[b]{2}{*}{$\begin{array}{c}\text { ODT/ppbV @ } \\
25^{\circ} \mathrm{C}\end{array}$} & \multicolumn{2}{|r|}{ Sensory } & \multicolumn{6}{|c|}{ SIFT-MS $^{1}$} \\
\hline & & Ref. $^{2}$ & Odor Note & Ref. $^{3}$ & $\mathrm{H}_{3} \mathrm{O}^{+}$ & $\mathrm{NO}^{+}$ & $\mathrm{O}_{2}{ }^{+}$ & Ref. $^{4}$ & $\mathrm{LOQ} / \mathrm{ppbV}$ \\
\hline Aldehydes & & & & & & & & & \\
\hline Formaldehyde & 490 & 1 & Acrid, suffocating & I & 31 & & & K & 6.6 \\
\hline Acetaldehyde & 50 & 5 & Fruit, apple & I & $45(63)$ & $43(61)$ & & K & 21.6 \\
\hline Propanal & 5.9 & 9 & Sweet, ester & IV & & 57 & & $\mathrm{~K}$ & 2.3 \\
\hline Butanal isomers & 0.34 & 1 & Rancid, sweaty + Fruit & I & & 71 & & $\mathrm{~K}, \mathrm{~L}$ & 1.1 \\
\hline Pentanal isomers & 0.45 & 9 & Almonds, pungent (pentanal) & II & & 85 & & $\mathrm{~L}, \mathrm{M}$ & 0.4 \\
\hline Hexanal & 0.83 & 10 & Grass & II & $\begin{array}{c}83,101 \\
(119)\end{array}$ & 99 & & $\mathrm{~K}$ & 1.1 \\
\hline Heptanal & 56 & 11 & Fresh, green, herbal & III & $115(133)$ & 113 & 96 & $\mathrm{~L}$ & 0.5 \\
\hline Nonanal & 40 & 12 & Citrus, rose & II & $143(161)$ & 141 & 124 & $\mathrm{~N}$ & 0.9 \\
\hline Decanal & 9.9 & 10 & $\begin{array}{l}\text { Soap, citrus, baking bread, } \\
\text { yeast, peanuts, burning }\end{array}$ & II & $157(175)$ & 155 & & $\mathrm{~L}$ & 0.5 \\
\hline Benzaldehyde & 140 & 10 & Almonds, bitter, musty, mushrooms & II & 107 & 105 & & $\mathrm{~K}$ & 0.5 \\
\hline Heptadienal & 13 & 13 & Green, pungent, fatty & III & 109 & & & $\mathrm{O}$ & 0.5 \\
\hline $\begin{array}{l}\text { Decadienal } \\
\text { Ketones }\end{array}$ & 0.026 & 14 & Citrus, fatty, oily, green, chicken & III & & 151 & 81 & $\mathrm{~L}$ & 0.5 \\
\hline Acetone & 42,500 & 1 & Fruit, sweet & I & & 88 & & K & 4.6 \\
\hline Butanone & 440 & 1 & Green apple, sweet, acetone-like & I, II & & 102 & & $\mathrm{~K}$ & 0.2 \\
\hline Pentanone isomers & 99 & 15 & Acetone-like, fruit & II & & 116 & & K & 0.5 \\
\hline $\begin{array}{l}\text { Methyl isobutyl } \\
\text { ketone }\end{array}$ & 170 & 1 & Sweet, acetone-like, minty & II & & 130 & & $\mathrm{P}$ & 0.5 \\
\hline Heptanone isomers & 6.9 & 1 & Soap & II & & 144 & & $\mathrm{Q}$ & 0.5 \\
\hline $\begin{array}{l}\text { 2,3-Butanedione } \\
\text { Alcohols }\end{array}$ & 0.051 & 1 & Butter & $\mathrm{V}$ & & 86 & & $\mathrm{~J}, \mathrm{~K}$ & 0.6 \\
\hline Ethanol & 530 & 1 & Sweet & $\mathrm{V}$ & $47(65)$ & $45(63)$ & & $\mathrm{R}$ & 68.5 \\
\hline Propanol isomers & 26,000 & 1 & Sharp musty & II & 43 & 59 & & $\mathrm{R}$ & 2.1 \\
\hline Butanol isomers & 220 & 1 & Alcoholic, sweet, banana & II & & 73 & & $\mathrm{R}$ & 4.0 \\
\hline Cresol isomers & 0.090 & 1 & Medicinal, urine, piggery, faecal/floral & II & & 108 & 108 & $S$ & 0.2 \\
\hline 2-Methylisoborneol & 0.0070 & 16 & Musty water, earthy & II & 151 & & & $\mathrm{~T}$ & 0.5 \\
\hline Geosmin & 0.0070 & 1 & Musty water, earthy & II & 165 & & & $\mathrm{O}$ & 0.5 \\
\hline
\end{tabular}


Table 4. Cont.

\begin{tabular}{|c|c|c|c|c|c|c|c|c|c|}
\hline \multirow[t]{2}{*}{ Compound } & \multirow[b]{2}{*}{$\begin{array}{c}\text { ODT/ppbV @ } \\
25^{\circ} \mathrm{C}\end{array}$} & \multicolumn{3}{|c|}{ Sensory } & \multicolumn{4}{|c|}{ SIFT-MS $^{1}$} & \multirow[b]{2}{*}{ LOQ/ppbV } \\
\hline & & Ref. $^{2}$ & Odor Note & Ref. $^{3}$ & $\mathrm{H}_{3} \mathrm{O}^{+}$ & $\mathrm{NO}^{+}$ & $\mathrm{O}_{2}{ }^{+}$ & Ref. ${ }^{4}$ & \\
\hline \multicolumn{10}{|l|}{$\begin{array}{c}\text { Monoterpenes and } \\
\text { derivatives }\end{array}$} \\
\hline Monoterpenes & 32 & 1 & Diverse odors; generally natural/fresh & II & & 136 & 136 & $\mathrm{U}$ & 0.5 \\
\hline Carvone & 5.5 & 17 & Mint, musty, oily & II & & 180 & & $\mathrm{~V}$ & 0.5 \\
\hline Dihydrocarvone & 170 & 18 & $\begin{array}{c}\text { Herb, mint, hospital, } \\
\text { sweet, wood, mushrooms }\end{array}$ & II & 153 & 182 & 152 & $\mathrm{~W}$ & 0.5 \\
\hline Thymol & 6.5 & 19 & $\begin{array}{l}\text { Thyme, aromatic, herbaceous, } \\
\text { chemical, lemongrass }\end{array}$ & II & & 150 & & $\mathrm{x}$ & 0.5 \\
\hline Hydrocarbons & & & & & & & & & \\
\hline Toluene & 340 & 1 & Rubber, musty, sweet, oily, solvent, gasoline & II & 93 & 92 & 92 & $\mathrm{Y}$ & 0.6 \\
\hline $\begin{array}{l}\text { Ethylbenzene + total } \\
\quad \text { xylene isomers }\end{array}$ & 170 & 1 & Sweet, aromatic & II & 107 & 106 & & Y & 0.6 \\
\hline $\mathrm{C}_{3}$-Alkylbenzenes & 170 & 1 & Aromatic, sweet & II & & 120 & 120 & $\mathrm{Y}$ & 0.5 \\
\hline $\begin{array}{c}\mathrm{C}_{4} \text {-Alkylbenzenes } \\
\text { (from } \\
\text { diethylbenzene) }\end{array}$ & 9.5 & 1 & Solvent, rubber, musty, oily & II & & 134 & 119 & $\mathrm{O}$ & 0.5 \\
\hline Decane & 620 & 1 & Gasoline & II & & 141 & 142 & $\mathrm{O}$ & 0.7 \\
\hline Undecane & 880 & 1 & Gasoline & II & & 155 & 156 & $\mathrm{O}$ & 0.5 \\
\hline Dodecane & 110 & 1 & Gasoline & II & & 169 & & $\mathrm{O}$ & 5.1 \\
\hline \multicolumn{10}{|l|}{ Chlorine-containing } \\
\hline Dichlorobenzene & 120 & 20 & Pleasant, aromatic & VI & 147 & 146 & 146 & $\mathrm{D}, \mathrm{N}$ & 0.5 \\
\hline $\begin{array}{l}\text { Chlorophenol } \\
\text { isomers }\end{array}$ & 0.19 & 21 & Medicinal & VII & 129 & & 128,130 & $\mathrm{O}$ & 0.5 \\
\hline
\end{tabular}

${ }^{1}$ SIFT-MS detection $\mathrm{m} / \mathrm{z}$ for reagent ions are shown in Daltons. ${ }^{2}$ ODT references: see Supplementary Data-Table 4 References. ${ }^{3}$ Odor note references: see Supplementary Data-Table 4 References. ${ }^{4}$ SIFT-MS library data references: see Supplementary Data-Table 4 References. 


\section{Results}

Tables 5-7 summarize the sensory panel and instrumental concentration data (in ppbV) for the influent and primary treatment, secondary liquids treatment, and secondary solids treatment, respectively. Due to the large number of discreet samples, the range of values for each treated odor source is presented here. The complete data set is available as Supplementary Materials. Variation within different sources may be attributed to many operational and environmental factors as analyzed further in the discussion.

Table 5. Sensory data and SIFT-MS concentration data (in ppbV) for odor sources from influent and primary treatment. The range of values for each source is shown. See the text and table footnotes for more details.

\begin{tabular}{|c|c|c|c|c|c|c|}
\hline & \multirow[b]{2}{*}{$\begin{array}{l}\mathrm{ODT} / \mathrm{ppbV} \\
\text { at } 25^{\circ} \mathrm{C}\end{array}$} & \multirow[b]{2}{*}{$\begin{array}{l}\text { Instrument } \\
\text { LOQ/ppbV }\end{array}$} & \multicolumn{4}{|c|}{ Odor Sources $^{1}$} \\
\hline & & & $\begin{array}{c}\text { Screens, } \\
\text { Primary Tanks, } \\
\text { IP-EF }\end{array}$ & $\begin{array}{l}\text { Screens } \\
\text { Building, } \\
\text { IP-SB }\end{array}$ & $\begin{array}{l}\text { Pumping } \\
\text { Station, } \\
\text { P-PS }\end{array}$ & $\begin{array}{c}\text { Splitter Box for } \\
\text { Reactor-Clarifiers, } \\
\text { P-SP }\end{array}$ \\
\hline Sample type & & & Biofilter & Ambient & Biofilter & Biofilter \\
\hline Number of samples & & & 13 & 2 & 2 & 6 \\
\hline \multicolumn{7}{|l|}{ Olfactometry } \\
\hline Concentration $^{2}$ & & & $65-41,086$ & 143-191 & $44-207$ & $55-4858$ \\
\hline Intensity ${ }^{3}$ & & & $\mathrm{I}, \mathrm{VL}, \mathrm{L}, \mathrm{M}, \mathrm{S}$ & I, VL & $\mathrm{I}, \mathrm{VL}$ & $\mathrm{VL}, \mathrm{L}, \mathrm{M}$ \\
\hline Character ${ }^{4}$ & & & V, Se, E, I, R, C & I, V & $\mathrm{I}, \mathrm{V}$ & $\mathrm{V}, \mathrm{C}, \mathrm{Se}, \mathrm{R}$ \\
\hline \multicolumn{7}{|l|}{ Odorants $^{5}$} \\
\hline \multicolumn{7}{|l|}{ Sulfur-containing } \\
\hline Hydrogen sulfide & 0.41 & 0.3 & $<$ LOQ-3800 & $4.2-4.4$ & $<$ LOQ-10 & $1.5-130$ \\
\hline Methyl mercaptan & 0.071 & 0.3 & $<$ LOQ-130 & $0.5-1.4$ & $0.6-0.7$ & $1.1-86$ \\
\hline $\begin{array}{l}\text { Dimethyl sulfide + } \\
\text { ethyl mercaptan }\end{array}$ & 3.0 & 0.5 & $<$ LOQ-7.9 [2] & $<$ LOQ & $<$ LOQ & $<$ LOQ-27 \\
\hline Dimethyl disulfide & 2.2 & 0.2 & $<$ LOQ-2.5 [4] & $<$ LOQ & $<$ LOQ-0.6 & $<\mathrm{LOQ}-30$ \\
\hline Dimethyl trisulfide & 1.5 & 0.5 & $<$ LOQ-15 [5] & $<$ LOQ-0.6 & $<$ LOQ- -8 & $<$ LOQ-6.9 \\
\hline Allyl methyl sulfide & 6.1 & 1.6 & $<\mathrm{LOQ}-4.6[2]$ & $<$ LOQ & $<$ LOQ & $<$ LOQ-5.9 \\
\hline Diethyl disulfide & 0.024 & 0.5 & $<\mathrm{LOQ}-1.1[1]$ & $<\mathrm{LOQ}$ & $<$ LOQ- 0.6 & $<$ LOQ-0.7 [1] \\
\hline Carbon disulfide & 210 & 7.9 & $<$ LOQ-13 [1] & $<$ LOQ-10.8 & $<$ LOQ & $<$ LOQ-73 \\
\hline Propyl mercaptans & 0.51 & 0.5 & $<$ LOQ-4.1 [4] & $<$ LOQ -0.9 & $<\mathrm{LOQ}$ & $<\mathrm{LOQ}-3.8$ \\
\hline Butyl mercaptans & 0.81 & 0.4 & $<\mathrm{LOQ}-1.5[3]$ & $<$ LOQ & $<$ LOQ-0.6 & $<$ LOQ -1.9 \\
\hline \multicolumn{7}{|l|}{ Nitrogen-containing } \\
\hline Ammonia & 1600 & 10.1 & $<$ LOQ-15 [2] & $<\mathrm{LOQ}$ & $<\mathrm{LOQ}$ & $<$ LOQ \\
\hline Methylamine & 36 & 1.6 & $<\mathrm{LOQ}-2.9[1]$ & $<\mathrm{LOQ}$ & $<$ LOQ & $<$ LOQ-3.4 \\
\hline Dimethylamine & 32 & 11.2 & $<$ LOQ & $<\mathrm{LOQ}$ & $<\mathrm{LOQ}$ & $<$ LOQ \\
\hline Trimethylamine & 2.4 & 2.3 & $<\mathrm{LOQ}$ & $3.8-7.5$ & $<\mathrm{LOQ}$ & $<\mathrm{LOQ}-4.1$ [2] \\
\hline Diethylamine & 47 & 7.6 & $<\mathrm{LOQ}$ & $<\mathrm{LOQ}$ & $<\mathrm{LOQ}$ & $<$ LOQ \\
\hline Triethylamine & 5.3 & 0.2 & $<\mathrm{LOQ}-0.5[3]$ & $<\mathrm{LOQ}$ & $<$ LOQ-0.5 & $<\mathrm{LOQ}-0.5$ [2] \\
\hline Isopropylamine & 25 & 3.5 & $<$ LOQ & $<$ LOQ-3.9 & $<$ LOQ & $<$ LOQ \\
\hline Putrescine & 2.8 & 2.0 & $<$ LOQ-72 [4] & $<\widehat{L O Q}$ & $<$ LOQ-5.7 & $<$ LOQ-78 \\
\hline Cadaverine & 2.4 & 1.3 & $<$ LOQ & $<\mathrm{LOQ}$ & $<\widehat{L O Q}$ & $<$ LOQ-2.8 [1] \\
\hline Indole & 0.29 & 0.2 & $<$ LOQ-0.3 [1] & $<\mathrm{LOQ}$ & $<$ LOQ & $<$ LOQ \\
\hline Skatole & 0.093 & 0.2 & $<$ LOQ & $<\mathrm{LOQ}$ & $<\mathrm{LOQ}$ & $<\mathrm{LOQ}-0.5$ [1] \\
\hline Pyridine & 62 & 0.2 & $<\mathrm{LOQ}$ & $<\mathrm{LOQ}$ & $<$ LOQ & $<\mathrm{LOQ}$ \\
\hline \multicolumn{7}{|l|}{ Volatile fatty acids } \\
\hline Acetic acid & 6.1 & 1.9 & $<\mathrm{LOQ}$ & $<$ LOQ & $<\mathrm{LOQ}$ & $<\mathrm{LOQ}-2.0[1]$ \\
\hline Butyric acid & 0.19 & 0.6 & $<\mathrm{LOQ}-1.1$ [2] & $<\mathrm{LOQ}$ & $<$ LOQ & $<\mathrm{LOQ}$ \\
\hline Valeric acid & 0.038 & 0.8 & $<$ LOQ-18 [5] & $<$ LOQ-0.8 & $<$ LOQ-1.2 & $0.9-17$ \\
\hline
\end{tabular}


Table 5. Cont.

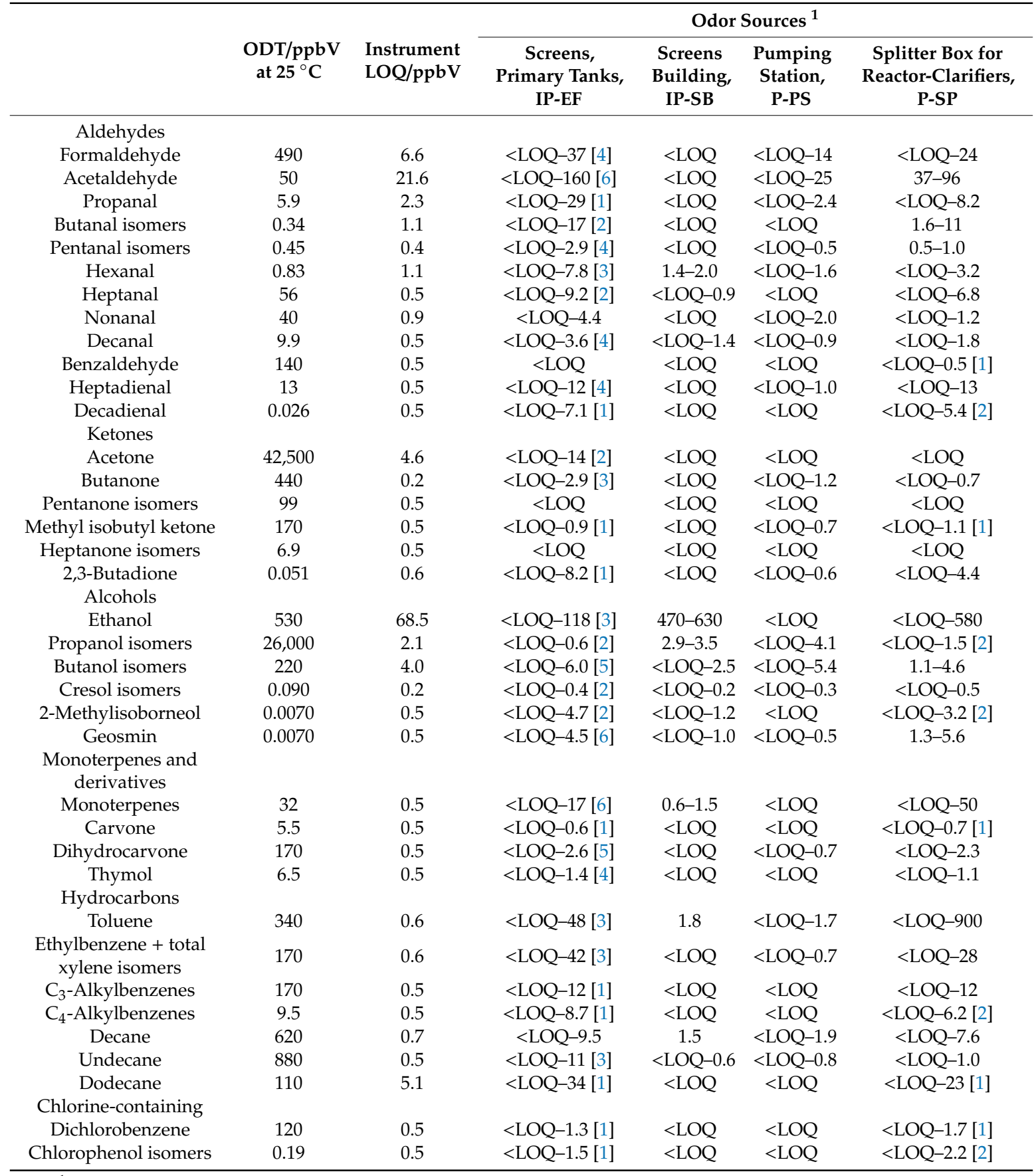

${ }^{1}$ For the SIFT-MS data, where less than $50 \%$ of samples report above the LOQ for a compound, the number of measurements greater than the LOQ is shown in square brackets after the concentration range. ${ }^{2}$ Odor concentration is in $\mathrm{OU} \mathrm{m}{ }^{-3} .{ }^{3}$ Odor intensity range, with most frequent in bold. Abbreviations: $\mathrm{I}=$ indiscernible, $\mathrm{VL}=$ very light, $\mathrm{L}=$ light, $\mathrm{M}=$ moderate, $\mathrm{S}=$ strong. ${ }^{4}$ Odor characters in order of high to low frequency. Abbreviations: $C=$ chemical,$E=$ earth,$F=$ rotten fish, $I=$ indiscernible,$M=$ musty, $R=$ rotten vegetation, $\mathrm{Se}=$ sewage, $\mathrm{V}=$ vegetation. ${ }^{5}$ Detected using SIFT-MS and converted to OAVs in ppbV, as described in the text. 
Table 6. Sensory data and SIFT-MS concentration data (in ppbV) for odor sources from secondary liquids treatment. The range of values for each source is shown. See the text and table footnotes for more details.

\begin{tabular}{|c|c|c|c|c|c|c|}
\hline & \multirow[b]{2}{*}{$\begin{array}{c}\text { ODT/ppbV } \\
\text { at } 25^{\circ} \mathrm{C}\end{array}$} & \multirow[b]{2}{*}{$\begin{array}{l}\text { Instrument } \\
\text { LOQ/ppbV }\end{array}$} & \multicolumn{4}{|c|}{ Odor Sources $^{1}$} \\
\hline & & & $\begin{array}{c}\text { Reactor } \\
\text { Aerobic-Location } \\
\text { 1, SW-A1 }\end{array}$ & $\begin{array}{c}\text { Reactor } \\
\text { Aerobic-Location } \\
\text { 2, SW-A2 }\end{array}$ & $\begin{array}{c}\text { Reactor } \\
\text { Anoxic-Location } \\
\text { 1, SW-AO }\end{array}$ & $\begin{array}{l}\text { Clarifier, } \\
\text { SW-CL }\end{array}$ \\
\hline Sample type & & & Flotation & Flotation & Flotation & Flotation \\
\hline Number of samples & & & 8 & 9 & 9 & 20 \\
\hline \multicolumn{7}{|l|}{ Olfactometry } \\
\hline Concentration $^{2}$ & & & $1551-50,370$ & $158-712$ & $210-890$ & $155-655$ \\
\hline Intensity $^{3}$ & & & M, S & $\mathrm{VL}, \mathrm{L}, \mathrm{M}$ & $\mathrm{L}, \mathrm{M}$ & $\mathrm{VL}, \mathrm{L}, \mathrm{M}$ \\
\hline Character ${ }^{4}$ & & & Se, E, V & $\mathrm{E}, \mathrm{V}, \mathrm{C}$ & $\mathrm{C}, \mathrm{V}$ & $\mathrm{V}, \mathrm{E}$ \\
\hline \multicolumn{7}{|l|}{ Odorants $^{5}$} \\
\hline \multicolumn{7}{|l|}{ Sulfur-containing } \\
\hline Hydrogen sulfide & 0.41 & 0.3 & $46-2100$ & $<\mathrm{LOQ}-2.3$ & $<$ LOQ-9.5 & $<$ LOQ-3.7 \\
\hline Methyl mercaptan & 0.071 & 0.3 & $34-190$ & $0.5-4.2$ & $<\mathrm{LOQ}-41$ & $<\mathrm{LOQ}-2.2$ \\
\hline $\begin{array}{l}\text { Dimethyl sulfide + } \\
\text { ethyl mercaptan }\end{array}$ & 3.0 & 0.5 & $2.9-9.3$ & $<$ LOQ-2.8 & $<$ LOQ-6.1 & $<\mathrm{LOQ}-2.7[3]$ \\
\hline Dimethyl disulfide & 2.2 & 0.2 & $0.4-1.1$ & $<$ LOQ -0.3 & $<$ LOQ -0.5 & $<\mathrm{LOQ}-0.7[9]$ \\
\hline Dimethyl trisulfide & 1.5 & 0.5 & $0.8-1.8$ & $<$ LOQ -2.9 & $<$ LOQ -13 & $<$ LOQ $-2.1[8]$ \\
\hline Allyl methyl sulfide & 6.1 & 1.6 & $<$ LOQ-8.2 & $<$ LOQ-3.6 [2] & $<$ LOQ-2.7 [3] & $<\mathrm{LOQ}-4.2[9]$ \\
\hline Diethyl disulfide & 0.024 & 0.5 & $<$ LOQ- $0.7[1]$ & $<$ LOQ & $<$ LOQ & $<$ LOQ $-0.5[1]$ \\
\hline Carbon disulfide & 210 & 7.9 & $<$ LOQ-14 [3] & $<$ LOQ-9.1 [1] & $<$ LOQ-9.8 [3] & $<\mathrm{LOQ}-11[3]$ \\
\hline Propyl mercaptans & 0.51 & 0.5 & $0.7-3.9$ & $<\mathrm{LOQ}-0.8$ [2] & $<\mathrm{LOQ}-1.1[3]$ & $<$ LOQ- $0.8[6]$ \\
\hline Butyl mercaptans & 0.81 & 0.4 & $1.1-1.4$ & $<$ LOQ -0.9 [2] & $<$ LOQ -1.1 & $<$ LOQ $-1.0[7]$ \\
\hline \multicolumn{7}{|l|}{ Nitrogen-containing } \\
\hline Ammonia & 1600 & 10.1 & $<\mathrm{LOQ}-17$ & $<$ LOQ-16 [2] & $<$ LOQ-22 [2] & $<\mathrm{LOQ}-25$ [2] \\
\hline Methylamine & 36 & 1.6 & $<$ LOQ-2.5 [2] & $<$ LOQ-5.7 [2] & $<\mathrm{LOQ}$ & $<\mathrm{LOQ}-2.6[4]$ \\
\hline Dimethylamine & 32 & 11.2 & $<$ LOQ & $<$ LOQ & $<\mathrm{LOQ}$ & $<$ LOQ \\
\hline Trimethylamine & 2.4 & 2.3 & $<$ LOQ-7.3 [3] & $<$ LOQ-9.3 [3] & $<$ LOQ-6.5 [3] & $<$ LOQ-4.9 [5] \\
\hline Diethylamine & 47 & 7.6 & $<$ LOQ & $<$ LOQ & $<$ LOQ & $<$ LOQ \\
\hline Triethylamine & 5.3 & 0.2 & $<$ LOQ- 0.7 & $<\mathrm{LOQ}$ & $<$ LOQ-0.4 [3] & $<$ LOQ-0.5 [8] \\
\hline Isopropylamine & 25 & 3.5 & $<$ LOQ & $<$ LOQ-6.1 [3] & $<$ LOQ-4.6 [2] & $<\mathrm{LOQ}-5.1[3]$ \\
\hline Putrescine & 2.8 & 2.0 & $<$ LOQ & $<$ LOQ-7.5 & $<$ LOQ -20 & $<$ LOQ-8.1 \\
\hline Cadaverine & 2.4 & 1.3 & $<$ LOQ-8.3 & $<$ LOQ-1.6 [1] & $<$ LOQ-2.6 [2] & $<\mathrm{LOQ}$ \\
\hline Indole & 0.29 & 0.2 & $<\mathrm{LOQ}-2.6$ & $<$ LOQ & $<$ LOQ & $<\mathrm{LOQ}-0.3$ [1] \\
\hline Skatole & 0.093 & 0.2 & $<$ LOQ & $<\mathrm{LOQ}$ & $<\mathrm{LOQ}$ & $<$ LOQ \\
\hline Pyridine & 62 & 0.2 & $<\mathrm{LOQ}$ & $<$ LOQ-0.3 [1] & $<\mathrm{LOQ}$ & $<\mathrm{LOQ}-0.2[2]$ \\
\hline \multicolumn{7}{|l|}{ Volatile fatty acids } \\
\hline Acetic acid & 6.1 & 1.9 & $<$ LOQ-2.5 [3] & $<$ LOQ-2.2 [1] & $<$ LOQ-5.3 [2] & $<\mathrm{LOQ}-3.6[2]$ \\
\hline Butyric acid & 0.19 & 0.6 & $<\mathrm{LOQ}-1.2$ & $<\mathrm{LOQ}-1.2$ [2] & $<\mathrm{LOQ}-0.7[3]$ & $<$ LOQ $-1.0[7]$ \\
\hline $\begin{array}{l}\text { Valeric acid } \\
\text { Aldehydes }\end{array}$ & 0.038 & 0.8 & $1.9-3.6$ & $<$ LOQ -2.5 & $<$ LOQ -7.8 & $<$ LOQ-2.6 \\
\hline Formaldehyde & 490 & 6.6 & $12-100$ & 9-52 & $<$ LOQ-47 & $<$ LOQ-57 \\
\hline Acetaldehyde & 50 & 21.6 & $82-310$ & $<$ LOQ-140 & $<$ LOQ-120 & $<$ LOQ-223 \\
\hline Propanal & 5.9 & 2.3 & $<$ LOQ-3.0 [1] & $<$ LOQ-4.1 [1] & $<$ LOQ-12 & $<\mathrm{LOQ}-3.3[1]$ \\
\hline Butanal isomers & 0.34 & 1.1 & $<$ LOQ -2.2 & $<$ LOQ-3.4 [4] & $<$ LOQ -5.5 & $<$ LOQ-3.0 [9] \\
\hline Pentanal isomers & 0.45 & 0.4 & $0.9-1.9$ & $<$ LOQ-0.8 & $0.7-2.3$ & $<\mathrm{LOQ}-1.4$ \\
\hline Hexanal & 0.83 & 1.1 & $1.2-3.4$ & $<\mathrm{LOQ}-2.5$ & $1.4-5.0$ & $<\mathrm{LOQ}-2.1$ \\
\hline Heptanal & 56 & 0.5 & $<$ LOQ-1.0 & $<\mathrm{LOQ}-1.9$ & $<$ LOQ-6.0 & $<\mathrm{LOQ}-1.1[3]$ \\
\hline Nonanal & 40 & 0.9 & $<$ LOQ -8.5 & $<$ LOQ-2.3 [4] & $<$ LOQ-2.3 [3] & $<$ LOQ-3.3 \\
\hline Decanal & 9.9 & 0.5 & $0.6-3.4$ & $<$ LOQ-3.0 & $<$ LOQ -3.3 & $<$ LOQ $-1.7[7]$ \\
\hline Benzaldehyde & 140 & 0.5 & $<$ LOQ-1.6 [2] & $<$ LOQ & $<$ LOQ & $<\mathrm{LOQ}-1.3[2]$ \\
\hline Heptadienal & 13 & 0.5 & $<\mathrm{LOQ}-1.5$ [2] & $<$ LOQ-2.9 [4] & $<$ LOQ-10.4 & $<$ LOQ-2.1 [8] \\
\hline $\begin{array}{l}\text { Decadienal } \\
\text { Ketones }\end{array}$ & 0.026 & 0.5 & $<$ LOQ & $<\mathrm{LOQ}-2.9$ [3] & $<\mathrm{LOQ}-7.9$ & $<\mathrm{LOQ}-1.1[1]$ \\
\hline Acetone & 42500 & 4.6 & $10-31$ & $<$ LOQ-8.2 [2] & $<$ LOQ-16 & $\begin{array}{c}<\mathrm{LOQ}-1200 \\
{[7]}\end{array}$ \\
\hline Butanone & 440 & 0.2 & $0.7-1.4$ & $<$ LOQ-0.6 [4] & <LOQ-1.6 & $<$ LOQ $-1.4[9]$ \\
\hline Pentanone isomers & 99 & 0.5 & $<$ LOQ & $<\mathrm{LOQ}$ & $<$ LOQ-2.1 [3] & $<$ LOQ \\
\hline $\begin{array}{l}\text { Methyl isobutyl } \\
\text { ketone }\end{array}$ & 170 & 0.5 & $<$ LOQ -8.3 & $<\mathrm{LOQ}-0.7$ [2] & $<\mathrm{LOQ}-0.9$ [3] & $<\mathrm{LOQ}-0.8[5]$ \\
\hline Heptanone isomers & 6.9 & 0.5 & $<$ LOQ & $<$ LOQ & $<$ LOQ & $<$ LOQ \\
\hline 2,3-Butadione & 0.051 & 0.6 & $<$ LOQ-1.6 & $<\mathrm{LOQ}-0.8$ [2] & $<$ LOQ-4.9 & $<$ LOQ-0.9 [4] \\
\hline
\end{tabular}


Table 6. Cont.

\begin{tabular}{|c|c|c|c|c|c|c|}
\hline & \multirow[b]{2}{*}{$\begin{array}{c}\mathrm{ODT} / \mathrm{ppbV} \\
\text { at } 25^{\circ} \mathrm{C}\end{array}$} & \multirow[b]{2}{*}{$\begin{array}{l}\text { Instrument } \\
\text { LOQ/ppbV }\end{array}$} & \multicolumn{4}{|c|}{ Odor Sources ${ }^{1}$} \\
\hline & & & $\begin{array}{c}\text { Reactor } \\
\text { Aerobic-Location } \\
\text { 1, SW-A1 }\end{array}$ & $\begin{array}{c}\text { Reactor } \\
\text { Aerobic-Location } \\
\text { 2, SW-A2 }\end{array}$ & $\begin{array}{c}\text { Reactor } \\
\text { Anoxic-Location } \\
\text { 1, SW-AO }\end{array}$ & $\begin{array}{l}\text { Clarifier, } \\
\text { SW-CL }\end{array}$ \\
\hline \multicolumn{7}{|l|}{ Alcohols } \\
\hline Ethanol & 530 & 68.5 & $<$ LOQ-250 & $<$ LOQ-2200 [3] & $<$ LOQ-420 [4] & $<$ LOQ-940 [7] \\
\hline Propanol isomers & 26,000 & 2.1 & $<$ LOQ-9.3 & $<$ LOQ -11 & $<$ LOQ-15 [4] & $<$ LOQ-4.1 [6] \\
\hline 2-Methylisoborneol & 0.0070 & 0.5 & $<$ LOQ & $<\mathrm{LOQ}-4.9$ [3] & $<$ LOQ-22 & $<$ LOQ-1.5 [2] \\
\hline Geosmin & 0.0070 & 0.5 & $0.6-1.6$ & $<\mathrm{LOQ}-5.4$ & $0.8-41$ & $<\mathrm{LOQ}-1.7$ \\
\hline \multicolumn{7}{|l|}{$\begin{array}{c}\text { Monoterpenes and } \\
\text { derivatives }\end{array}$} \\
\hline Monoterpenes & 32 & 0.5 & $<$ LOQ-2.8 & $<\mathrm{LOQ}-2.6$ & $0.8-8.9$ & $<$ LOQ-1.6 [6] \\
\hline Toluene & 340 & 0.6 & $0.8-170$ & $<\mathrm{LOQ}-2.0$ & $<$ LOQ-2.5 & $<$ LOQ-2.1 [5] \\
\hline $\begin{array}{l}\text { Ethylbenzene }+ \\
\text { total xylene isomers }\end{array}$ & 170 & 0.6 & $<$ LOQ-13 & $<$ LOQ-1.8 [4] & $<$ LOQ-4.3 & $<$ LOQ-1.2 [4] \\
\hline $\mathrm{C}_{3}$-Alkylbenzenes & 170 & 0.5 & $<\mathrm{LOQ}-2.2[3]$ & $<$ LOQ-0.9 [1] & $<$ LOQ-5.4 & $<$ LOQ-1.0 [1] \\
\hline $\mathrm{C}_{4}$-Alkylbenzenes & 9.5 & 0.5 & $<\mathrm{LOQ}-1.2[2]$ & $<$ LOQ-1.3 [1] & $<$ LOQ-6.5 & $<$ LOQ-1.2 [1] \\
\hline Decane & 620 & 0.7 & $<$ LOQ -3.6 & $<\mathrm{LOQ}-1.9[1]$ & $1.5-28$ & $<$ LOQ-3.4 [8] \\
\hline Undecane & 880 & 0.5 & $<$ LOQ-0.9 & $<\mathrm{LOQ}-2.0[4]$ & $<$ LOQ-32 & $<$ LOQ-3.4 [3] \\
\hline Dodecane & 110 & 5.1 & $<\mathrm{LOQ}-8.8$ [2] & $<$ LOQ-9.9 [1] & $<$ LOQ-160 & $<\mathrm{LOQ}-10$ [4] \\
\hline \multicolumn{7}{|l|}{ Chlorine-containing } \\
\hline Dichlorobenzene & 120 & 0.5 & $<\mathrm{LOQ}$ & $<\mathrm{LOQ}$ & $<$ LOQ-1.3 [1] & $<\mathrm{LOQ}$ \\
\hline $\begin{array}{l}\text { Chlorophenol } \\
\text { isomers }\end{array}$ & 0.19 & 0.5 & $<$ LOQ & $<$ LOQ & $<\mathrm{LOQ}-1.6$ [2] & $<$ LOQ-1.4 [1] \\
\hline
\end{tabular}

${ }^{1}$ For the SIFT-MS data, where less than 50\% of samples report above the LOQ for a compound, the number of measurements greater than the LOQ is shown in square brackets after the concentration range. ${ }^{2}$ Odor concentration is in $\mathrm{OU} \mathrm{m}{ }^{-3} \cdot{ }^{3}$ Odor intensity range, with most frequent in bold. Abbreviations: $\mathrm{I}=$ indiscernible, $\mathrm{VL}=$ very light, $\mathrm{L}=$ light, $\mathrm{M}=$ moderate, $\mathrm{S}=$ strong. ${ }^{4}$ Odor characters in order of high to low frequency. Abbreviations: $\mathrm{C}=$ chemical, $\mathrm{E}=$ earth, $\mathrm{F}=$ rotten fish, $\mathrm{I}=$ indiscernible, $\mathrm{M}=$ musty, $\mathrm{R}=$ rotten vegetation, $\mathrm{Se}=$ sewage, $\mathrm{V}=$ vegetation. ${ }^{5}$ Detected using SIFT-MS and converted to OAVs in ppbV, as described in the text. 
Table 7. Sensory data and SIFT-MS concentration data (in ppbV) for odor sources from secondary solids treatment. The range of values for each source is shown. See the text and table footnotes for more details.

\begin{tabular}{|c|c|c|c|c|c|c|c|c|c|c|}
\hline & \multirow[b]{2}{*}{$\begin{array}{l}\mathrm{ODT} / \mathrm{ppbV} \\
\text { at } 25^{\circ} \mathrm{C}\end{array}$} & \multirow[b]{2}{*}{$\begin{array}{c}\text { Inst. } \\
\text { LOQ/ppbV }\end{array}$} & \multicolumn{8}{|c|}{ Odor Sources $^{1}$} \\
\hline & & & $\begin{array}{c}\text { Gravity } \\
\text { Thickeners, } \\
\text { SS-GT }\end{array}$ & $\begin{array}{l}\text { Dissolved Air } \\
\text { Flotation, } \\
\text { SS-AF }\end{array}$ & $\begin{array}{l}\text { Digester Feed, } \\
\text { SS-DF }\end{array}$ & $\begin{array}{c}\text { Gas Turbine } \\
\text { Stack, SS-TS }{ }^{2}\end{array}$ & $\begin{array}{l}\text { Centrifu., } \\
\text { SS-CE }\end{array}$ & $\begin{array}{c}\text { Biosolids } \\
\text { Storage, } \\
\text { SS-BS }\end{array}$ & $\begin{array}{c}\text { Ammonia } \\
\text { Scrubber, } \\
\text { SS-AS }\end{array}$ & $\begin{array}{c}\text { Sludge Stack, } \\
\text { SS-SS }\end{array}$ \\
\hline Sample type & & & Biofilter & Flotation & Biofilter & Stack & Biofilter & Biofilter & Biofilter & Stack \\
\hline Number of samples & & & 5 & 6 & 3 & 3 & 3 & 10 & 4 & 4 \\
\hline \multicolumn{11}{|l|}{ Olfactometry } \\
\hline Concentration $^{3}$ & & & $24-153$ & $138-11205$ & $107-157$ & $570-4567$ & $98-710$ & $119-269$ & $204-3661$ & $301-3244$ \\
\hline Intensity ${ }^{4}$ & & & I, L & $\mathrm{L}, \mathrm{M}, \mathrm{S}$ & $\mathrm{VL}, \mathrm{L}$ & $\mathrm{L}, \mathrm{S}$ & $\mathrm{VL}, \mathrm{L}, \mathrm{S}$ & $\mathrm{VL}, \mathrm{L}$ & $\mathrm{L}, \mathrm{M}$ & $\mathrm{L}, \mathrm{M}, \mathrm{S}$ \\
\hline Character $^{5}$ & & & $\mathrm{I}, \mathrm{Se}, \mathrm{V}$ & E, V, R, M, Se, C & $\mathrm{V}$ & $\mathrm{C}$ & $\mathrm{C}, \mathrm{E}, \mathrm{V}$ & $\mathrm{V}, \mathrm{E}$ & E, V, R, C & Se, C, V, F \\
\hline \multicolumn{11}{|l|}{ Odorants ${ }^{6}$} \\
\hline Sulfur-containing & & & & & & & & & & \\
\hline Hydrogen sulfide & 0.41 & 0.3 & $<$ LOQ-3.4 & $<$ LOQ-0.7 [2] & $1.4-7.1$ & $1-2-3.9$ & $<$ LOQ-1.4 & $<$ LOQ-3.1 & $<\mathrm{LOQ}-13$ & $1.0-34$ \\
\hline Methyl mercaptan & 0.071 & 0.3 & $<$ LOQ -1.3 & $<$ LOQ-160 & $0.5-0.8$ & $0.9-1.6{ }^{*}$ & $0.4-2.0$ & $<$ LOQ-1.7 & $1.2-2.4$ & $<$ LOQ -8.2 \\
\hline $\begin{array}{l}\text { Dimethyl sulfide }+ \\
\text { ethyl mercaptan }\end{array}$ & 3.0 & 0.5 & $<\mathrm{LOQ}-0.5[1]$ & $<\mathrm{LOQ}-10[2]$ & $<$ LOQ -0.6 & $<\mathrm{LOQ}, *$ & $<$ LOQ-0.7 [1] & $<\mathrm{LOQ}-0.8$ [2] & $<$ LOQ-1.7 & $<\mathrm{LOQ}$ \\
\hline Dimethyl disulfide & 2.2 & 0.2 & $<$ LOQ-0.5 [2] & $<\mathrm{LOQ}-7.7$ [2] & $<$ LOQ-0.3 [1] & $<\mathrm{LOQ}, *$ & $<\mathrm{LOQ}-0.4$ & $<$ LOQ & $<$ LOQ -1.2 & $<$ LOQ-1.7 \\
\hline Dimethyl trisulfide & 1.5 & 0.5 & $<$ LOQ-1.1 [2] & $<\mathrm{LOQ}-2.1$ & $<$ LOQ-1.2 [1] & $<$ LOQ-0.3, [1] & $<$ LOQ-0.9 [1] & $<\mathrm{LOQ}-0.6[3]$ & $<$ LOQ-1.8 & $<$ LOQ-0.8 \\
\hline Allyl methyl sulfide & 6.1 & 1.6 & $<$ LOQ & $<\mathrm{LOQ}-2.3$ & $<$ LOQ-2.5 [1] & $<\mathrm{LOQ}-2.3^{*}{ }^{*}$ & $<$ LOQ-1.8 [1] & $<$ LOQ & $<$ LOQ-2.2 [1] & $<$ LOQ \\
\hline Diethyl disulfide & 0.024 & 0.5 & $<$ LOQ-0.5 [1] & $<$ LOQ-1.0 [1] & $<$ LOQ & $<\mathrm{LOQ}, *$ & $<$ LOQ & $<$ LOQ & $<$ LOQ -0.7 [1] & $<\mathrm{LOQ}$ \\
\hline Carbon disulfide & 210 & 7.9 & $<$ LOQ-11.4 [1] & $<$ LOQ-14 [1] & $<\mathrm{LOQ}$ & $<\mathrm{LOQ}, *$ & $<$ LOQ & $<\mathrm{LOQ}$ & $<$ LOQ & $<$ LOQ \\
\hline Propyl mercaptans & 0.51 & 0.5 & $<\mathrm{LOQ}-0.8$ [2] & $<\mathrm{LOQ}-1.2[1]$ & $<\mathrm{LOQ}$ & $<\mathrm{LOQ}, *$ & $<$ LOQ-0.6 [1] & $<\mathrm{LOQ}-1.2$ & $<$ LOQ-0.6 [1] & $<\mathrm{LOQ}-1.6$ \\
\hline Butyl mercaptans & 0.81 & 0.4 & $<\mathrm{LOQ}-0.5[1]$ & $<$ LOQ-1.9 [2] & $<$ LOQ-1.0 [1] & $<\mathrm{LOQ}-1.8, *$ & $<\mathrm{LOQ}-0.9$ & $<$ LOQ-0.5 [3] & $<$ LOQQ-0.9 & $<$ LOQQ \\
\hline \multicolumn{11}{|l|}{ Nitrogen-containing } \\
\hline Ammonia & 1600 & 10.1 & $<\mathrm{LOQ}$ & $<$ LOQ & $<$ LOQ & $<$ LOQ-12 & $11-16$ & $<$ LOQ-15 [2] & $\begin{array}{c}<\mathrm{LOQ}-3700 \\
{[1]}\end{array}$ & $<$ LOQ-4700 \\
\hline Methylamine & 36 & 1.6 & $<$ LOQ & $<$ LOQ-1.8 [1] & $<$ LOQ-1.6 [1] & $<$ LOQ-5.3,* & $<$ LOQ-1.8 & $<$ LOQ-3.5 [4] & $<$ LOQ -3.0 & $<$ LOQ-4.7 \\
\hline Dimethylamine & 32 & 11.2 & $<\mathrm{LOQ}$ & $<\mathrm{LOQ}-20[1]$ & $<$ LOQ & $<\mathrm{LOQ}, *$ & $<$ LOQ & $<$ LOQ & $<$ LOQ-26 [1] & $<$ LOQ-11.9 [1] \\
\hline Trimethylamine & 2.4 & 2.3 & $<\mathrm{LOQ}$ & $<$ LOQ & $<$ LOQ-2.9 & $<$ LOQ & $<$ LOQ-6.4 & $<\mathrm{LOQ}-38$ [4] & $<\mathrm{LOQ}-4.8$ & $<$ LOQ-30.1 \\
\hline Diethylamine & 47 & 7.6 & $<$ LOQ & $<$ LOQ & $<\mathrm{LOQ}$ & $<\mathrm{LOQ},{ }^{*}$ & $<\mathrm{LOQ}$ & $<$ LOQ & $<$ LOQ-23 [1] & $<\mathrm{LOQ}-8.6$ [1] \\
\hline Triethylamine & 5.3 & 0.2 & $<$ LOQ -0.3 [2] & $<$ LOQ -0.2 [2] & $<\mathrm{LOQ}$ & $<$ LOQ-1.5 [1] & $<$ LOQ-0.3 & $<\mathrm{LOQ}-0.2[2]$ & $<$ LOQ -0.3 & $<\mathrm{LOQ}-0.2[1]$ \\
\hline Isopropylamine & 25 & 3.5 & $<$ LOQ & $<$ LOQ & $<$ LOQ & $<\mathrm{LOQ},{ }^{*}$ & $<$ LOQ & $<\mathrm{LOQ}-4.6[3]$ & $<$ LOQ-76 [1] & $<$ LOQ-79 \\
\hline Putrescine & 2.8 & 2.0 & $<$ LOQ-7.3 [2] & $<$ LOQ-2.4 [1] & $<$ LOQ-5.5 [1] & $<\mathrm{LOQ}-2.1, *$ & $2.7-3.9$ & $<\mathrm{LOQ}-2.6[3]$ & $<\mathrm{LOQ}-11.2$ & $<$ LOQ-2.1 [1] \\
\hline Cadaverine & 2.4 & 1.3 & $<$ LOQ & $<\mathrm{LOQ}-1.6[1]$ & $<$ LOQ & $<\mathrm{LOQ}, *$ & $<$ LOQ & $<$ LOQ & $<$ LOQ & $<$ LOQ \\
\hline Indole & 0.29 & 0.2 & $<$ LOQ & $<$ LOQ & $<$ LOQ & $<$ LOQ-0.5 [1] & $<$ LOQ & $<\mathrm{LOQ}$ & $<$ LOQ & $<$ LOQ \\
\hline Skatole & 0.093 & 0.2 & $<$ LOQ & $<\mathrm{LOQ}$ & $<\mathrm{LOQ}$ & $<\mathrm{LOQ}, *$ & $<\mathrm{LOQ}-0.2[1]$ & $<\mathrm{LOQ}$ & $<\mathrm{LOQ}$ & $<$ LOQ \\
\hline Pyridine & 62 & 0.2 & $<$ LOQ & $<\mathrm{LOQ}-1.6$ [2] & $<$ LOQ & $<\mathrm{LOQ}, *$ & $<\mathrm{LOQ}$ & $<\mathrm{LOQ}$ & $<$ LOQ-0.2 [1] & $<$ LOQ \\
\hline
\end{tabular}


Table 7. Cont.

\begin{tabular}{|c|c|c|c|c|c|c|c|c|c|c|}
\hline & \multirow[b]{2}{*}{$\begin{array}{l}\mathrm{ODT} / \mathrm{ppbV} \\
\text { at } 25^{\circ} \mathrm{C}\end{array}$} & \multirow[b]{2}{*}{$\begin{array}{c}\text { Inst. } \\
\text { LOQ/ppbV }\end{array}$} & \multicolumn{8}{|c|}{ Odor Sources $^{1}$} \\
\hline & & & $\begin{array}{l}\text { Gravity } \\
\text { Thickeners, } \\
\text { SS-GT }\end{array}$ & $\begin{array}{l}\text { Dissolved Air } \\
\text { Flotation, } \\
\text { SS-AF }\end{array}$ & $\begin{array}{l}\text { Digester Feed, } \\
\text { SS-DF }\end{array}$ & $\begin{array}{c}\text { Gas Turbine } \\
\text { Stack, SS-TS }{ }^{2}\end{array}$ & $\begin{array}{l}\text { Centrifu., } \\
\text { SS-CE }\end{array}$ & $\begin{array}{l}\text { Biosolids } \\
\text { Storage, } \\
\text { SS-BS }\end{array}$ & $\begin{array}{c}\text { Ammonia } \\
\text { Scrubber, } \\
\text { SS-AS }\end{array}$ & $\begin{array}{l}\text { Sludge Stack, } \\
\text { SS-SS }\end{array}$ \\
\hline \multicolumn{11}{|l|}{ Volatile fatty acids } \\
\hline Acetic acid & 6.1 & 1.9 & $<\mathrm{LOQ}$ & $<$ LOQ-5.4 [1] & $<$ LOQ-2.5 [1] & $<$ LOQ-31 & $<\mathrm{LOQ}-4.1$ & $<$ LOQ-5.0 & $<$ LOQ & $<$ LOQ-4.2 \\
\hline Butyric acid & 0.19 & 0.6 & $<$ LOQ & $<$ LOQ-0.8 [1] & $<$ LOQ & $<$ LOQ-1.3 [1] & $<$ LOQ & $<$ LOQ & $<$ LOQ-0.6 [1] & $<\mathrm{LOQ}$ \\
\hline $\begin{array}{l}\text { Valeric acid } \\
\text { Aldehydes }\end{array}$ & 0.038 & 0.8 & $<$ LOQ-1.6 [2] & $<$ LOQ-1.6 [2] & $<$ LOQ-1.9 & $1.0-10.7$ & $1.5-2.5$ & $<$ LOQ-1.0 & $<$ LOQ-3.2 & $<\mathrm{LOQ}$ \\
\hline Formaldehyde & 490 & 6.6 & $<\mathrm{LOQ}-23$ & $<$ LOQ-110 & $19-28$ & $37-4400$ & $<$ LOQ -32 & $<\mathrm{LOQ}-63$ & $13-35$ & $<$ LOQ-32 \\
\hline Acetaldehyde & 50 & 21.6 & $<$ LOQ -83 & $<$ LOQ-100 & $55-128$ & $<$ LOQ-1100 & $<$ LOQ-98 & $<$ LOQ-138 & $<$ LOQ-98 & $<$ LOQ-58 \\
\hline Propanal & 5.9 & 2.3 & $<$ LOQ & $<$ LOQ & $<\mathrm{LOQ}$ & 19-124 & $<$ LOQ & $<$ LOQ & $<$ LOQ & $<\mathrm{LOQ}-3$ [1] \\
\hline Butanal isomers & 0.34 & 1.1 & $<$ LOQ-1.4 [1] & $<$ LOQ-1.3 [1] & $<$ LOQ & $<$ LOQ-16 & $<$ LOQ-2.6 & $<$ LOQ-2.4 & $<$ LOQ-3.2 & $<$ LOQ-1.6 [1] \\
\hline Pentanal isomers & 0.45 & 0.4 & $<\mathrm{LOQ}-0.8$ [2] & $<$ LOQ-0.7 [2] & $<$ LOQ-0.9 & $0.8-8.5$ & $0.7-0.9$ & $<\mathrm{LOQ}-0.7[4]$ & $<\mathrm{LOQ}-1.5$ & $<$ LOQ $-0.4[1]$ \\
\hline Hexanal & 0.83 & 1.1 & $<$ LOQ-1.9 [1] & $<$ LOQ-2.0 & $<$ LOQ-1.9 [1] & $<$ LOQ-6.8 [1] & $<\mathrm{LOQ}-2.0$ & $<$ LOQ-1.4 [3] & $<\mathrm{LOQ}-2.6$ & $<\mathrm{LOQ}-1.6[1]$ \\
\hline Heptanal & 56 & 0.5 & $<$ LOQ & $<\mathrm{LOQ}-0.7$ & $<$ LOQ & $<$ LOQ-1.0 [1] & $<\mathrm{LOQ}-0.7$ [2] & $<\mathrm{LOQ}-0.8$ [2] & $<$ LOQ-1.9 [1] & $<$ LOQ \\
\hline Nonanal & 40 & 0.9 & $<$ LOQ-1.4 [2] & $<$ LOQ-2.5 [2] & $<$ LOQ-2.6 [1] & $<$ LOQ-1.5 [1] & $<\mathrm{LOQ}-2.1[1]$ & $<$ LOQ-1.5 [4] & $<$ LOQ-1.8 & $<$ LOQ-1.2 [1] \\
\hline Decanal & 9.9 & 0.5 & $<$ LOQ & $<$ LOQ-1.1 [2] & $<$ LOQ-0.8 [1] & $<$ LOQ-3.3 [1] & $<$ LOQ-1.2 & $<\mathrm{LOQ}-0.7[1]$ & $<$ LOQ-2.2 & $<\mathrm{LOQ}-0.5[1]$ \\
\hline Benzaldehyde & 140 & 0.5 & $<\mathrm{LOQ}$ & $<$ LOQ & $<$ LOQ & $<\mathrm{LOQ}-4.9[1]$ & $<$ LOQ & $<\mathrm{LOQ}-0.5[1]$ & $<$ LOQ-1.6 [1] & $<$ LOQ \\
\hline Heptadienal & 13 & 0.5 & $<$ LOQ-1.8 [2] & $<$ LOQ-2.2 & $<\mathrm{LOQ}$ & $1.4-15$ & $<\mathrm{LOQ}-2.6[1]$ & $<\mathrm{LOQ}-1.6[3]$ & $<$ LOQ & $<$ LOQ-2.5 [1] \\
\hline $\begin{array}{l}\text { Decadienal } \\
\text { Ketones }\end{array}$ & 0.026 & 0.5 & $<$ LOQ & $<$ LOQ & $<\mathrm{LOQ}$ & $<\mathrm{LOQ}$ & $<$ LOQ-1.1 [1] & $<$ LOQ & $<\mathrm{LOQ}$ & $<$ LOQ \\
\hline Acetone & 42500 & 4.6 & $<\mathrm{LOQ}$ & $<$ LOQ-8.4 [2] & $<$ LOQ-5.6 [1] & $4.7-49$ & $<\mathrm{LOQ}$ & $<\mathrm{LOQ}$ & $<$ LOQ -18 & $<$ LOQ \\
\hline Butanone & 440 & 0.2 & $<$ LOQ-0.4 [2] & $<$ LOQ-0.4 [1] & $<$ LOQ-0.6 & $0.5-2.6$ & $<\mathrm{LOQ}-0.4$ & $<\mathrm{LOQ}-0.4$ [2] & $<$ LOQ-2.9 & $<$ LOQ-0.6 \\
\hline Pentanone isomers & 99 & 0.5 & $<$ LOQ-1.1 [1] & $<\mathrm{LOQ}-0.5$ [1] & $<\mathrm{LOQ}$ & $<\mathrm{LOQ}$ & $<\mathrm{LOQ}$ & $<$ LOQ & $<\mathrm{LOQ}$ & $<$ LOQ \\
\hline $\begin{array}{l}\text { Methyl isobutyl } \\
\text { ketone }\end{array}$ & 170 & 0.5 & $<\mathrm{LOQ}$ & $<$ LOQ-0.6 [1] & $<$ LOQ-0.6 [1] & $<$ LOQ & $<\mathrm{LOQ}-0.7$ [1] & $<\mathrm{LOQ}-0.7$ [1] & $<$ LOQ0.7 [1] & $<\mathrm{LOQ}$ \\
\hline Heptanone isomers & 6.9 & 0.5 & $<$ LOQ & $<\mathrm{LOQ}$ & $<$ LOQ & $<$ LOQ & $<\mathrm{LOQ}$ & $<\mathrm{LOQ}$ & $<$ LOQ & $<$ LOQ \\
\hline $\begin{array}{l}\text { 2,3-Butadione } \\
\text { Alcohols }\end{array}$ & 0.051 & 0.6 & $<\mathrm{LOQ}$ & $<\mathrm{LOQ}-1.3$ [2] & $<$ LOQ & $0.6-45$ & $<$ LOQ-0.6 [1] & $<\mathrm{LOQ}$ & $<$ LOQ-1.3 & $<\mathrm{LOQ}-0.6$ [1] \\
\hline Ethanol & 530 & 68.5 & $<\mathrm{LOQ}$ & $<\mathrm{LOQ}-430$ [2] & $<$ LOQ-88 [1] & $<\mathrm{LOQ}$ & $<$ LOQ-200 & $<$ LOQ-970 [3] & $<$ LOQ-70 [1] & $<$ LOQ-870 [1] \\
\hline Propanol isomers & 26000 & 2.1 & $<$ LOQ & $<$ LOQ & $<$ LOQ-6.0 [1] & $<\mathrm{LOQ}$ & $2.9-4.7$ & $<\mathrm{LOQ}-61$ & $<\mathrm{LOQ}-8.6$ & $<$ LOQ-16 \\
\hline Butanol isomers & 220 & 4.0 & $<$ LOQ-4.9 [2] & $<$ LOQ-3.0 [2] & $1.4-2.9$ & $2.1-3.5$ & $<\mathrm{LOQ}-3.3$ & $<\mathrm{LOQ}-2.2[3]$ & $<$ LOQ-15 & $<$ LOQ-2.3 [1] \\
\hline Cresol isomers & 0.090 & 0.2 & $<$ LOQ-0.3 [1] & $<$ LOQ-0.7 [2] & $<$ LOQ & $<$ LOQ-5.5 [1] & $<$ LOQ- 0.2 [1] & $<$ LOQ-0.3 [1] & $<$ LOQ-0.6 & $<$ LOQ \\
\hline 2-Methylisoborneol & 0.0070 & 0.5 & $<$ LOQ & $<$ LOQ & $<$ LOQ & $<$ LOQ-1.1 [1] & $<$ LOQ & $<\mathrm{LOQ}$ & $<$ LOQ-1.6 & $<\mathrm{LOQ}$ \\
\hline Geosmin & 0.0070 & 0.5 & $<\mathrm{LOQ}-0.9$ [2] & $<$ LOQ-2.2 [1] & $<$ LOQ- 0.7 & $<$ LOQ-1.1 [1] & $0.9-1.8$ & $<$ LOQ-0.7 [1] & $1.0-2.1$ & $<\mathrm{LOQ}$ \\
\hline
\end{tabular}


Table 7. Cont.

\begin{tabular}{|c|c|c|c|c|c|c|c|c|c|c|}
\hline & \multirow[b]{2}{*}{$\begin{array}{l}\mathrm{ODT} / \mathrm{ppbV} \\
\text { at } 25^{\circ} \mathrm{C}\end{array}$} & \multirow[b]{2}{*}{$\begin{array}{c}\text { Inst. } \\
\mathrm{LOQ} / \mathrm{ppbV}\end{array}$} & \multicolumn{8}{|c|}{ Odor Sources $^{1}$} \\
\hline & & & $\begin{array}{l}\text { Gravity } \\
\text { Thickeners, } \\
\text { SS-GT }\end{array}$ & $\begin{array}{l}\text { Dissolved Air } \\
\text { Flotation, } \\
\text { SS-AF }\end{array}$ & $\begin{array}{l}\text { Digester Feed, } \\
\text { SS-DF }\end{array}$ & $\begin{array}{l}\text { Gas Turbine } \\
\text { Stack, SS-TS }{ }^{2}\end{array}$ & $\begin{array}{l}\text { Centrifu., } \\
\text { SS-CE }\end{array}$ & $\begin{array}{c}\text { Biosolids } \\
\text { Storage, } \\
\text { SS-BS }\end{array}$ & $\begin{array}{c}\text { Ammonia } \\
\text { Scrubber, } \\
\text { SS-AS }\end{array}$ & $\begin{array}{l}\text { Sludge Stack, } \\
\text { SS-SS }\end{array}$ \\
\hline \multicolumn{11}{|l|}{$\begin{array}{l}\text { Monoterpenes and } \\
\text { derivatives }\end{array}$} \\
\hline Monoterpenes & 32 & 0.5 & $<$ LOQ -0.9 & $<$ LOQ & $<$ LOQ -0.7 & $<$ LOQ & $0.6-5.1$ & $<$ LOQ-1.0 & $<$ LOQ-1.1 & $0.5-1.1$ \\
\hline Carvone & 5.5 & 0.5 & $<$ LOQ & $<\mathrm{LOQ}$ & $<$ LOQ & $<\mathrm{LOQ}$ & $<\mathrm{LOQ}$ & $<$ LOQ & $<$ LOQQ & $<\mathrm{LOQ}$ \\
\hline Dihydrocarvone & 170 & 0.5 & $<$ LOQ-1.6 [2] & $<$ LOQ-0.8 [1] & $<$ LOQ-0.9 [1] & $<$ LOQ & $0.8-1.0$ & $<\mathrm{LOQ}-0.7$ [2] & $<$ LOQ-1.3 & $<\mathrm{LOQ}$ \\
\hline Thymol & 6.5 & 0.5 & $<$ LOQ-0.6 [2] & $<$ LOQ & $<$ LOQ-0.9 [1] & $<\mathrm{LOQ}-6.5[1]$ & $<$ LOQ-0.9 & $<$ LOQ & $<$ LOQ & $<\mathrm{LOQ}$ \\
\hline Hydrocarbons & & & & & & & & & & \\
\hline Toluene & 340 & 0.6 & $<$ LOQ-17 [1] & <LOQ-1.1 [1] & $<\mathrm{LOQ}-1.0[1]$ & $<$ LOQ-11 [1] & $<$ LOQ-3.4 & $<$ LOQ-2.7 & $<$ LOQ-2.9 & $<$ LOQ-1.4 \\
\hline $\begin{array}{l}\text { Ethylbenzene + total } \\
\text { xylene isomers }\end{array}$ & 170 & 0.6 & $<$ LOQ-1.3 [1] & $<$ LOQ-0.7 [1] & $<$ LOQ & $<$ LOQ-2.8 [1] & $<$ LOQ-1.0 [1] & $<$ LOQ-0.8 [1] & $<$ LOQ-2.3 & $<$ LOQ \\
\hline $\mathrm{C}_{3}$-Alkylbenzenes & 170 & 0.5 & $<\mathrm{LOQ}$ & $<\mathrm{LOQ}$ & $<\mathrm{LOQ}$ & $<$ LOQ-3.2 [1] & $<$ LOQ-1.1 & $<$ LOQ-0.6 [1] & $<$ LOQ-2.0 & $<$ LOQ-3.0 \\
\hline $\mathrm{C}_{4}$-Alkylbenzenes & 9.5 & 0.5 & $<\mathrm{LOQ}$ & $<\mathrm{LOQ}$ & $<\mathrm{LOQ}$ & $<$ LOQ-2.1 [1] & $<$ LOQ-1.6 & $<$ LOQ & $<$ LOQ-1.7 & $<$ LOQ-1.2 [1] \\
\hline Decane & 620 & 0.7 & $<\mathrm{LOQ}-1.8[2]$ & $<$ LOQ $-1.1[1]$ & $<$ LOQ-1.8 [1] & $<$ LOQ-4.6 [1] & $1.1-3.1$ & $<$ LOQ-1.7 & $<$ LOQ -2.8 & $<\mathrm{LOQ}-1.2$ \\
\hline Undecane & 880 & 0.5 & $<$ LOQ-0.9 [1] & $<$ LOQ & $<\mathrm{LOQ}-0.8[1]$ & $<$ LOQ-3.8 & $<$ LOQ-2.7 & $<\mathrm{LOQ}-1.3$ [2] & $<$ LOQ-2.0 [1] & $<$ LOQ-1.1 \\
\hline Dodecane & 110 & 5.1 & $<$ LOQ & $<$ LOQ-7.9 [1] & $<$ LOQ & $<$ LOQQ & $<\mathrm{LOQ}-10$ & $<$ LOQ & $<$ LOQ & $<$ LOQ \\
\hline Chlorine-containing & & & & $<$ LOQ & & & & & & \\
\hline Dichlorobenzene & 120 & 0.5 & $<$ LOQ & $<\mathrm{LOQ}$ & $<$ LOQ & $<$ LOQ & $<$ LOQ & $<$ LOQ & $<\mathrm{LOQ}$ & $<\mathrm{LOQ}$ \\
\hline Chlorophenol isomers & 0.19 & 0.5 & $<\mathrm{LOQ}$ & $<\mathrm{LOQ}$ & $<\mathrm{LOQ}$ & $<\mathrm{LOQ}-4.8[1]$ & $<\mathrm{LOQ}$ & $<$ LOQ-1.1 [1] & $<\mathrm{LOQ}$ & $<\mathrm{LOQ}$ \\
\hline
\end{tabular}

${ }^{1}$ For the SIFT-MS data, where less than $50 \%$ of samples report above the LOQ for a compound, the number of measurements greater than the LOQ is shown in square brackets after the concentration range. ${ }^{2}$ For sample SS-TS-3, the combination of high levels of incomplete combustion products and moisture prevented quantitation of some odorants. These are indicated by an asterisk $(*) .{ }^{3}$ Odor concentration is in $\mathrm{OU} \mathrm{m}{ }^{-3} .{ }^{4}$ Odor intensity range, with most frequent in bold. Abbreviations: $\mathrm{I}=$ indiscernible, $\mathrm{VL}=\mathrm{very}$ light, $\mathrm{L}=$ light, $\mathrm{M}=\mathrm{moderate}$, $\mathrm{S}=$ strong. ${ }^{5}$ Odor characters in order of high to low frequency. Abbreviations: $\mathrm{C}=$ chemical, $\mathrm{E}=$ earth, $\mathrm{F}=$ rotten fish, $\mathrm{I}=$ indiscernible, $\mathrm{M}=$ musty, $\mathrm{R}=$ rotten vegetation, Se $=$ sewage, $\mathrm{V}=$ vegetation. ${ }^{6}$ Detected using SIFT-MS and converted to OAVs in ppbV, as described in the text. 
In each of Tables 5-7, the literature ODTs and instrumental LOQs are repeated to simplify comparison. Sensory characters (odor notes and intensities) are shown in abbreviated form and described in the table footnotes. Odor and instrumental concentrations are shown as observed ranges (in ppbV). For the SIFT-MS data, where less than 50\% of samples report above the LOQ for a compound, the number of measurements greater than the LOQ is shown in square brackets after the concentration range.

Figure 3 graphs represent a variety of odor sources throughout the treatment process and their associated sampling approaches. The chemical species variability within the same source was displayed along with the associated odor threshold value. Note that values less than the SIFT-MS LOQ are not shown. The ODT of each compound was indicated using a dotted red line. The sensory data (Figure 4) corresponding to these odor sources (Figure 3) showed similar individual sample variability for many reasons discussed later. There was variability in these representative sources of odor notes (Figure 5). In Figure 5, a frequency of unity is assigned where a single note is recorded, while each note was assigned a frequency of one-half when two are recorded.

To facilitate comparison of instrumental concentration data with sensory data, the concentrations were converted to OAVs using published odor detection thresholds. Only compounds with significant odor activities were further investigated. Hydrogen sulfide and methyl mercaptan were dominant in most odor sources. The odor compounds were grouped together in terms of primary odor categories defined by Fisher et al. [2]. Table 8 summarizes the key odorants, the primary odor category to which they have been assigned, and their correlation to the odor wheel of Fisher et al. [2] with the odor notes from the sensory analysis conducted here. Note that the characteristic "earthy/musty" odor notes for wastewater may be attributed to the presence of geosmin and 2-methylisoborneol [35]. Due to their very low ODTs (lower than the instrumental LOQ) and the complexity of the volatile emissions from the wastewater matrix, these compounds could not be detected and quantified reliably using SIFT-MS, so are omitted from the analysis. Fisher et al. [2] were unable to detect these compounds with GC/MS due to the very low ODTs. These compounds may still influence the odor character and olfactometry concentration as the method is designed to report the minimum odor threshold concentration.

Figure 6 shows the odor composition determined using SIFT-MS for each odor source, together with the total OAV obtained from the sum of the key odorants. Mean concentrations of different source samples were used (a zero value was used when the SIFT-MS measurement was $<$ LOQ) as a representation of that source. This was performed due to discreet sample variability at the same source. The range of total OAVs for the source is noted under the mean to indicate spread. In contrast to the concentration-based figures, the sum of hydrogen sulfide and methyl mercaptan OAVs dominated the odor for many sources.

Interestingly, the hydrogen sulfide ratio reduces during the wastewater treatment process. Hydrogen sulfide is high in the screens and primary sewage samples. The liquid process then moves through an 8-zone alternating anoxic/aerobic step-feed reactor process to the clarifier. A reduction in the ratio of hydrogen sulfide can be seen along this secondary treatment process along with a reduction of the OAV.

The total OAV for each sample was evaluated for its correlation with the odor concentration determined by the sensory panel. Figure 7 provides a comparison of the odor concentration determined by dilution olfactometry and the total odor activity determined using SIFT-MS. There is something of a trend as indicated by the dashed line.

The results in Figure 6 suggest that methyl mercaptan may contribute more to the odor impact at different stages of the process than hydrogen sulfide, the most frequently measured odor indicator. Humans are more sensitive to methyl mercaptan than hydrogen sulfide based on their ODTs (Table 4). SIFT-MS quantifies hydrogen sulfide and methyl mercaptan with particularly high degrees of confidence, except when formaldehyde concentrations are elevated, and the sample is very moist (the gas turbine stack sample SS-TS-3 is the only occurrence in the present study). The results presented here suggest that hydrogen sulfide measurement does not serve as a surrogate measure of methyl mercaptan, 
as shown in Figure 8. This figure summarizes data for all samples in which both hydrogen sulfide and methyl mercaptan OAVs could be determined (i.e., they are greater than the instrumental LOQ).

(a)

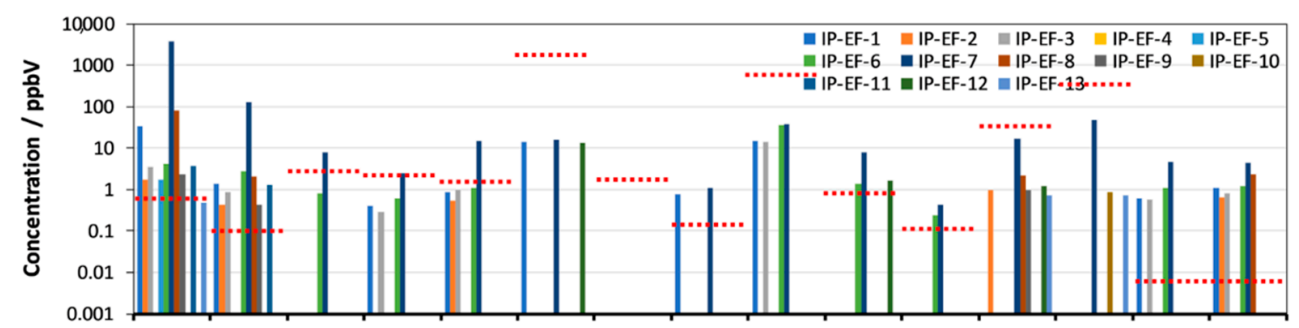

(b)
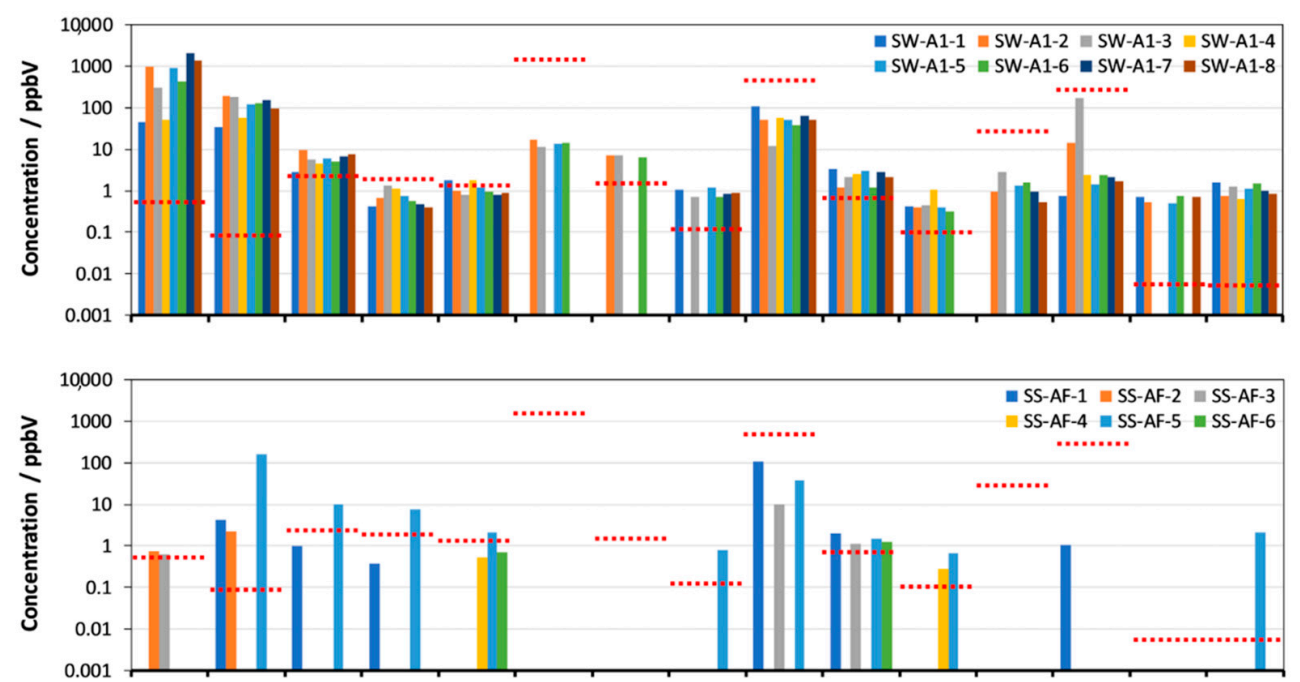

(d)
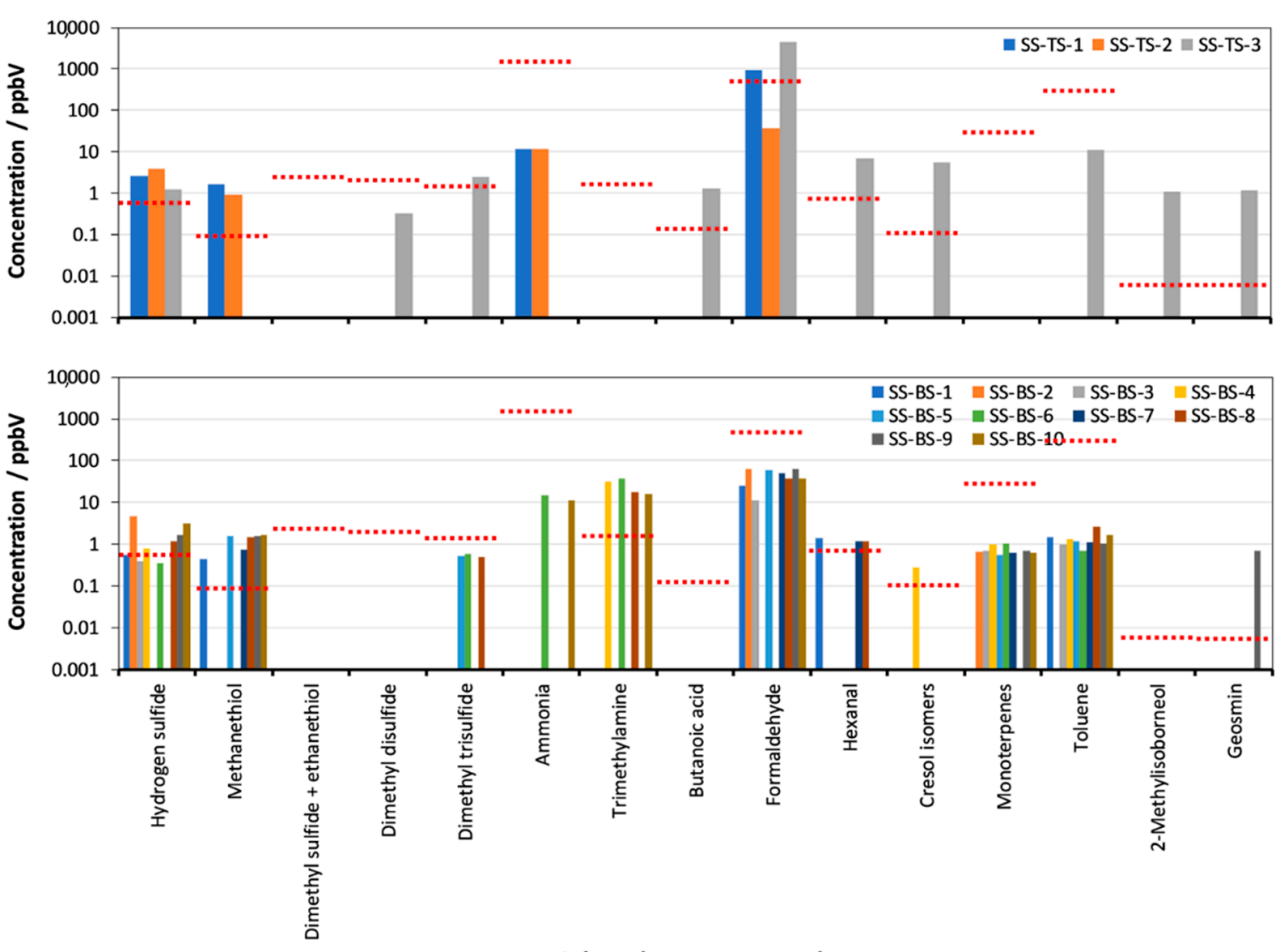

Selected target compounds

Figure 3. SIFT-MS concentration data (in ppbV on a logarithmic scale) for more significant odorants from a variety of odor sources: (a) influent biofilters, (b) reactor aerobic-location 1, (c) dissolved air flotation, (d) gas turbine stacks, and (e) biofilter at the biosolids storage location. ODTs are indicated approximately with dotted red lines. 


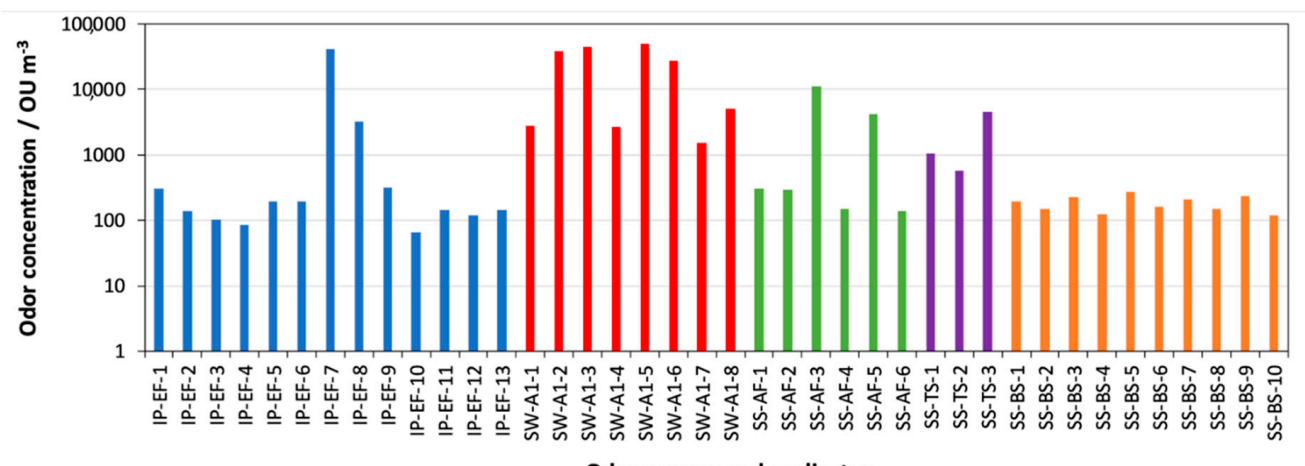

Odor sources and replicates

Figure 4. Odor concentrations (in OU $\mathrm{m}^{-3}$ on a logarithmic scale) for selected sources (Figure 3) determined using dynamic dilution olfactometry.

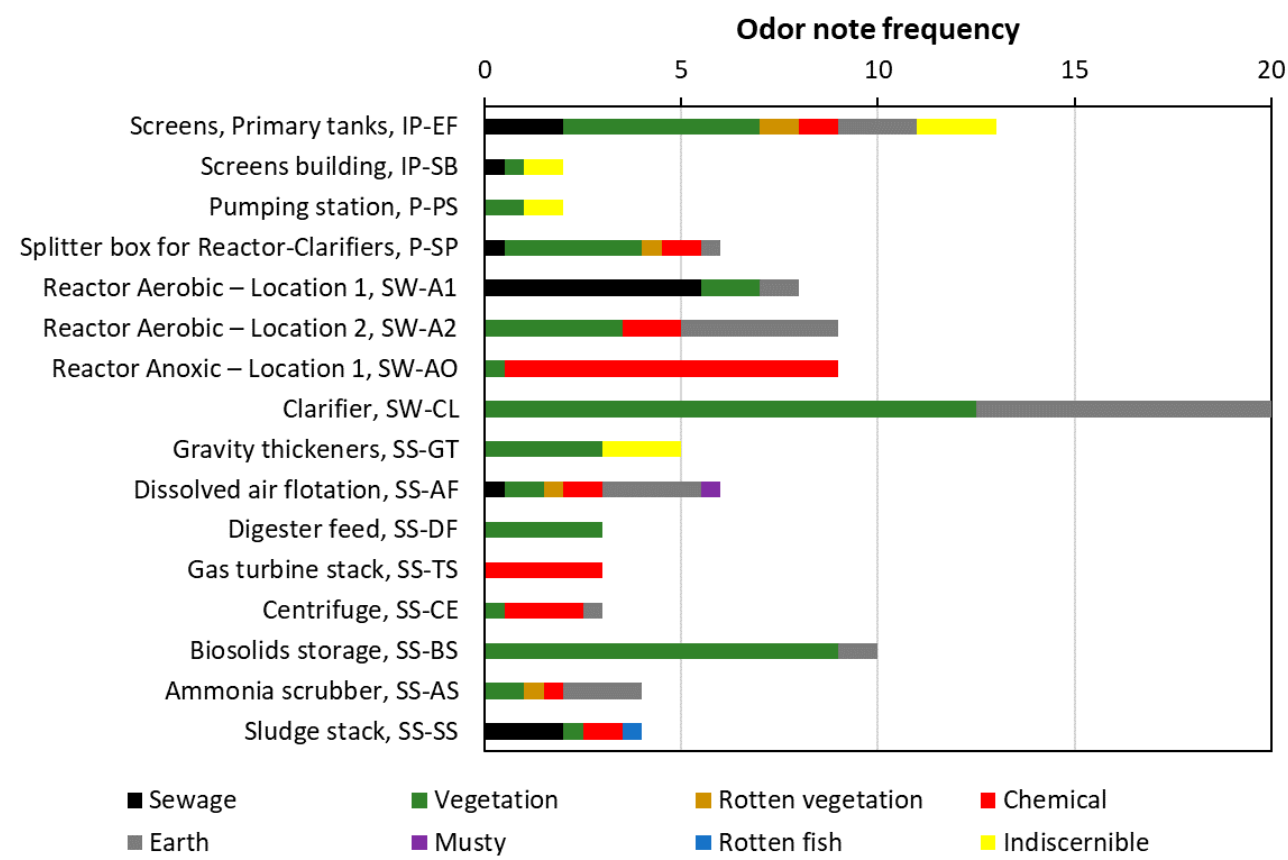

Figure 5. Odor notes assigned by the sensory panel. A frequency of 1 is used if only one odor note was assigned, while 0.5 is assigned to each note if two notes were assigned to a single sample. The frequency will always equal 1 per sampling occasion.

Table 8. Primary odor categories for wastewater, their correlation with the sensory panel's informal odor notes, and the most significant odor-active compounds detected using SIFT-MS.

\begin{tabular}{|c|c|c|}
\hline Primary Odor Category $^{1}$ & $\begin{array}{l}\text { Sensory Panel Lexicon } \\
\text { Oodor Note }{ }^{2}\end{array}$ & Compounds Included $(*=$ Not Included in Total) \\
\hline Earthy/musty & Earth, musty & Not considered $^{3}$ \\
\hline Fecal/manure & Sewage & Cresol isomers \\
\hline Fishy/ammonia & Rotten fish & Ammonia, isopropylamine, trimethylamine \\
\hline Natural/fresh & Vegetation & Pentanal isomers, hexanal, monoterpenes \\
\hline Rancid/putrid & - & Butyric acid, butanal isomers \\
\hline Solvent & Chemical & $\begin{array}{l}\text { Formaldehyde, acetaldehyde, propanal, ethanol, } \\
\text { toluene, } \mathrm{C}_{4} \text {-alkylbenzenes }\end{array}$ \\
\hline Sulfur & Sewage, rotten vegetation & $\begin{array}{l}\text { *Hydrogen sulfide, }{ }^{*} \text { methyl mercaptan, dimethyl } \\
\text { sulfide + ethanethiol, dimethyl disulfide, dimethyl } \\
\text { trisulfide, diethyl disulfide, propyl mercaptans } \\
\text { (total), butyl mercaptans (total) }\end{array}$ \\
\hline
\end{tabular}

\footnotetext{
${ }^{1}$ From Reference [2]. ${ }^{2}$ From sensory panel results (see Methods section). ${ }^{3}$ For reasons described in the text, compounds in this class are not detectable in wastewater using SIFT-MS.
} 

三 Hydrogen sulfide
IIII Methyl mercaptan
- Other organosulfur
Fishy/ammonia
s Solvent
:Rancid/putrid
Natural/fresh
8 Faecal/manure

Screens, Primary tanks, Screens building, IP-SB

Pumping station, P-PS

Splitter box for Reactor-
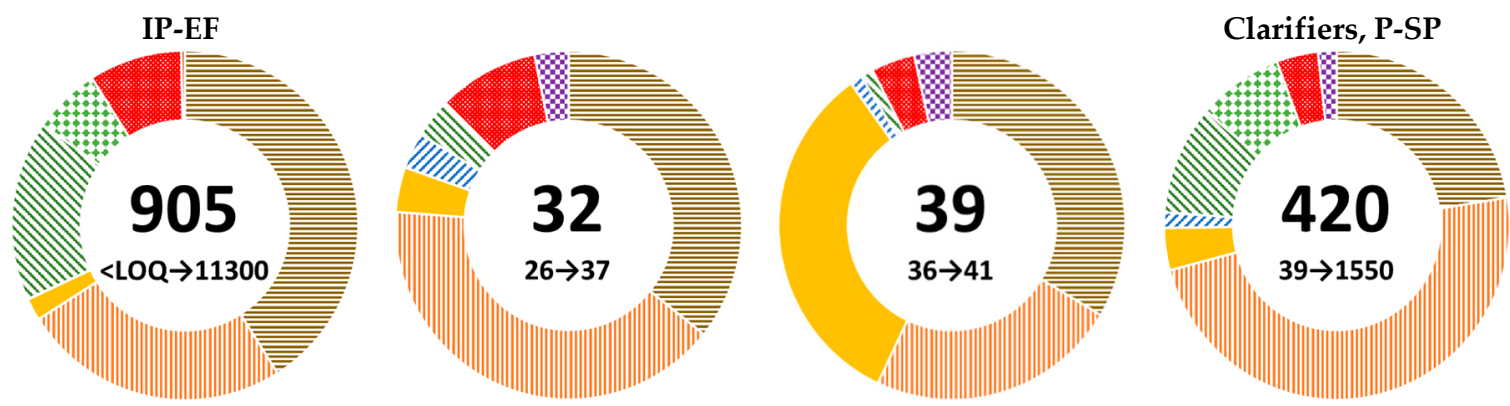

Reactor Aerobic-Location Reactor Aerobic-Location Reactor Anoxic-Location

\section{Clarifier, SW-CL}
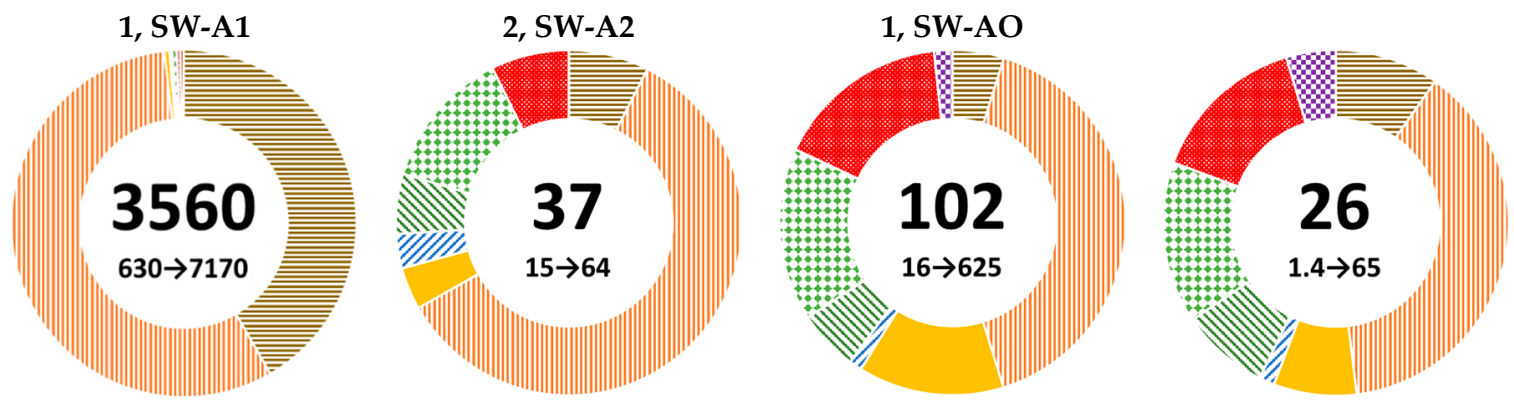

Gravity thickeners, SS-

Dissolved air flotation,

Digester feed, SS-DF

Gas turbine stack, SS-
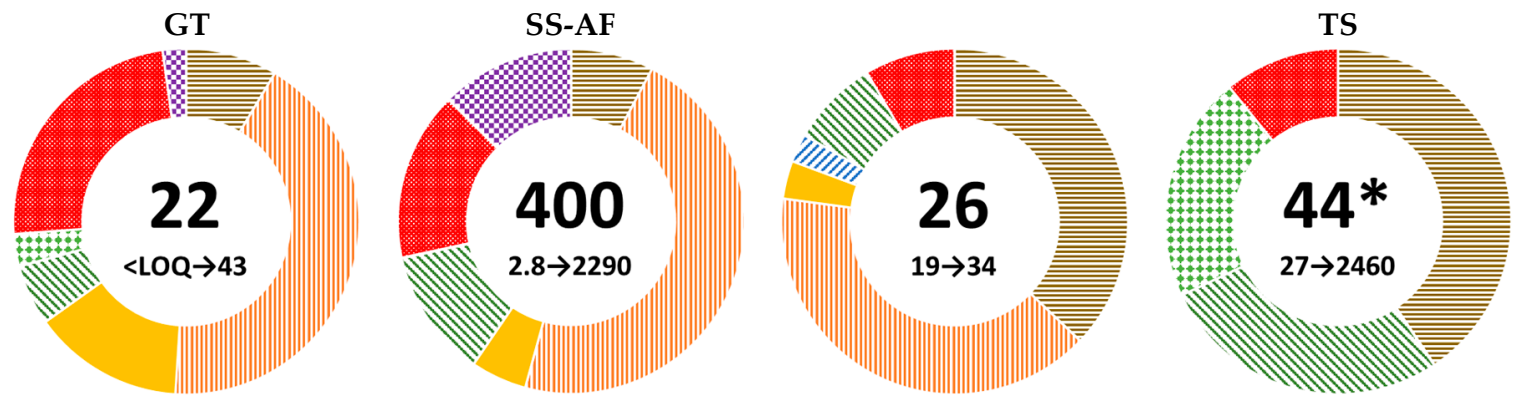

Centrifuge, SS-CE

Biosolids storage, SS-BS

Ammonia scrubber, SS-

Sludge stack, SS-SS
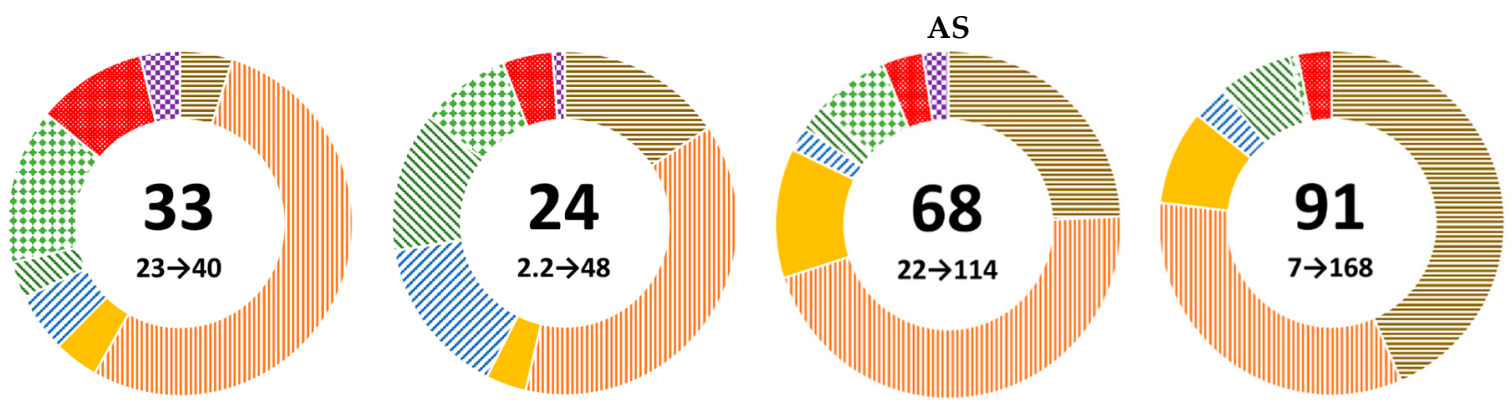

Figure 6. Dominant odorants/groups of odorants with similar notes for each odor source as a proportion of total odor activity measured using SIFT-MS. The large number in the center of each chart is the total OAV for the individual samples averaged across the samples of that type (except for the outlier in one of the gas turbine stack samples*). The small numbers show the range of total OAVs. *For the gas turbine, the outlier was eliminated since many sulfur compounds were not measurable. 


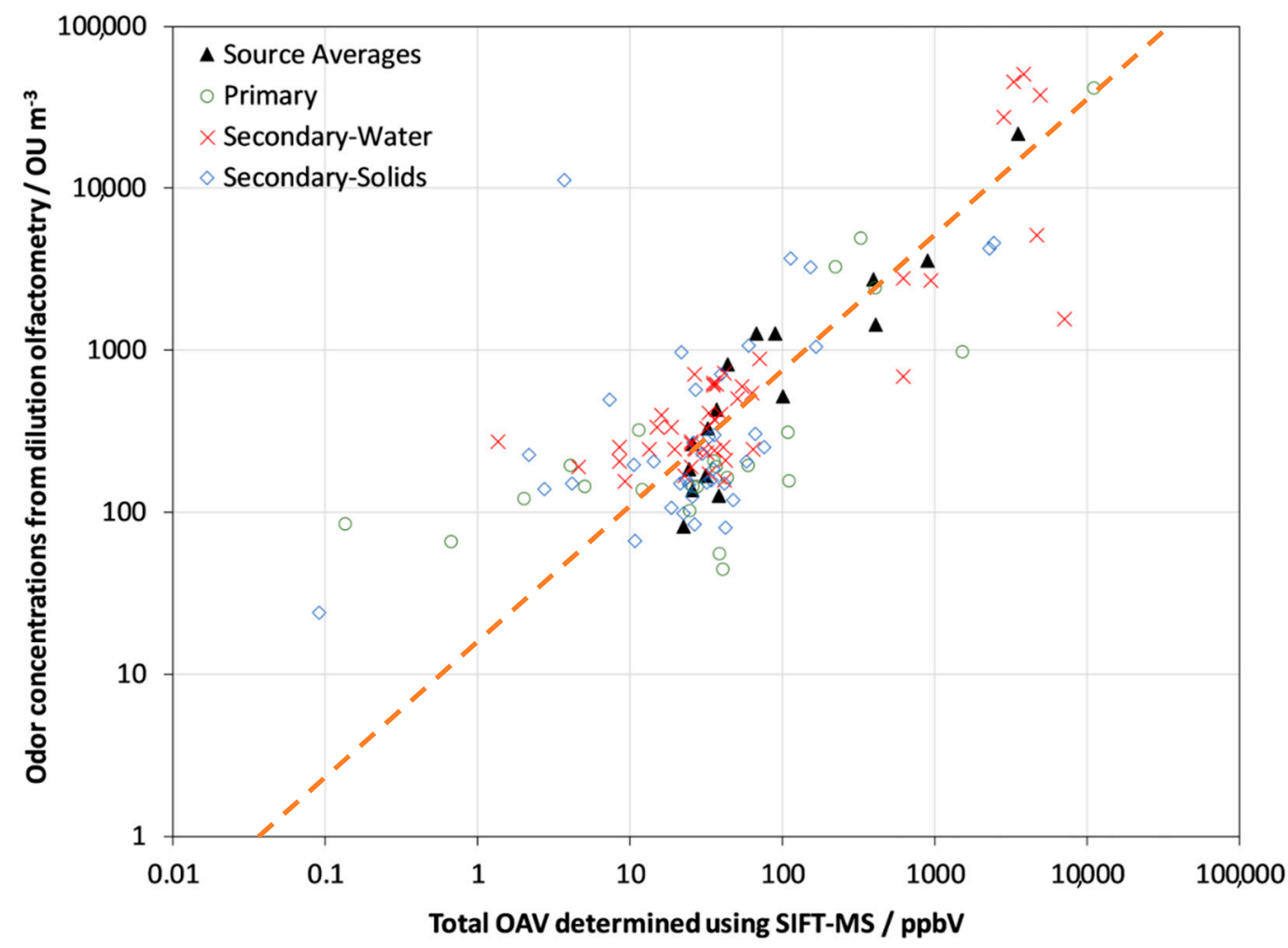

Figure 7. Odor concentrations from dilution olfactometry and SIFT-MS. Data for (a) primary, (b) secondary water, and (c) secondary solids odor sources are shown in different colors. Averaged values across all samples for a given odor source are shown with black triangles $\left(R^{2}=0.985\right.$; slope 5.91$)$.

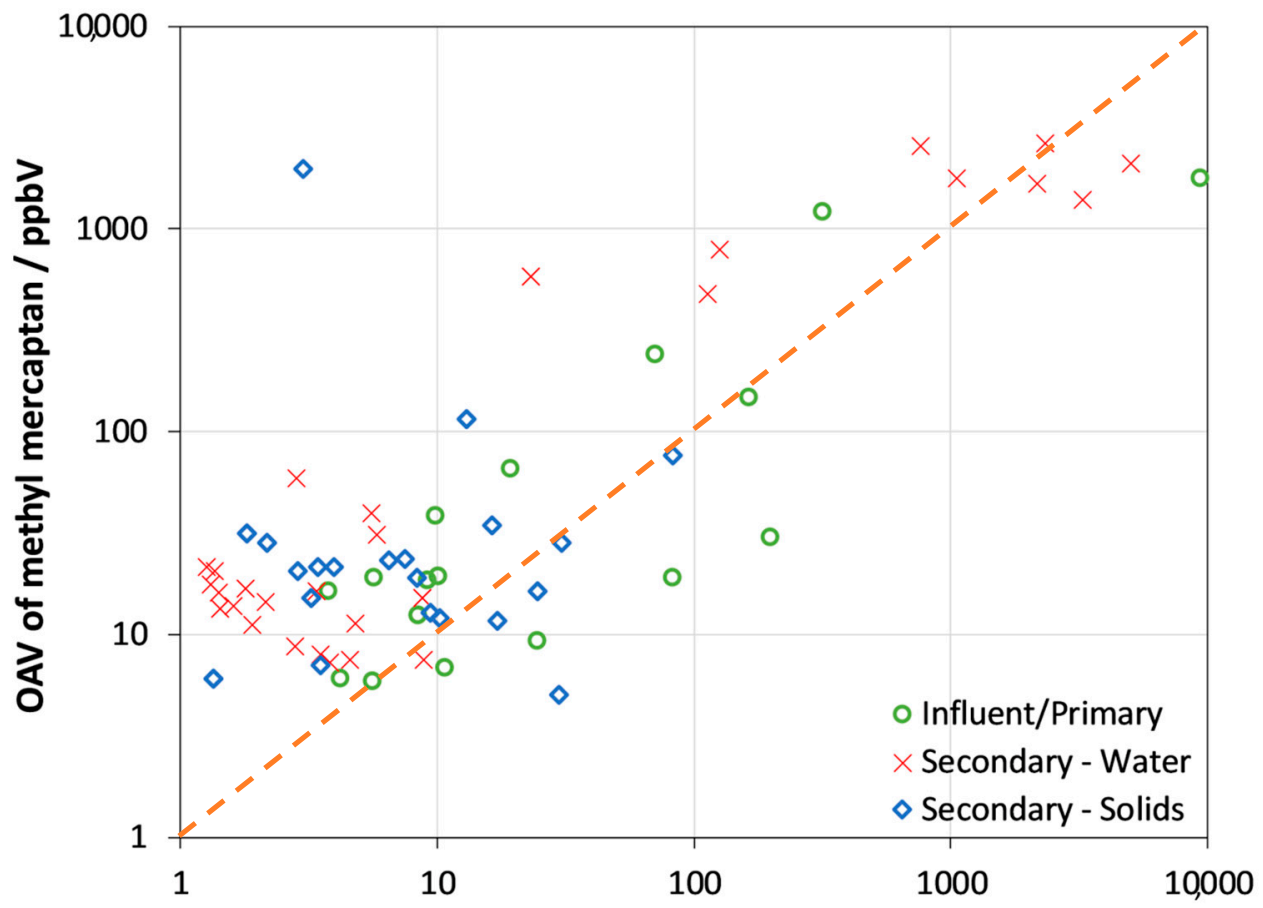

OAV of hydrogen sulfide / ppbV

Figure 8. The relationship between the dominant sulfur odorants (hydrogen sulfide and methyl mercaptan) observed in the WWTP using SIFT-MS for the primary (green circles), secondary water (red crosses), and secondary solids (blue diamonds) odor sources. Note the logarithmic axes. 


\section{Discussion}

The purpose of this paper is to evaluate the potential of SIFT-MS for instrumental analysis of odorants in WWTPs through comparison with the literature and with sensory results obtained for the same samples. The aim of the ongoing two-yearly odor emissions study at the plant was to give an overview of odor emissions after odor mitigation systems have removed most of the odor from the raw source.

Each set of samples for each site were collected under potentially different operational and environmental conditions. These fluctuating conditions contribute to the variation seen at each site both in the olfactometry and SIFT-MS data. Another contributing factor to the observed variation is the location on the odor bed in which the samples were taken. Each location may have odor bed media of differing efficiency and compaction condition. Under dry conditions and without sufficient coverage of the sprinkler system (see Table 9 for an indication of rainfall conditions over the course of the study), media may dry out and short-circuits in the filter bed may form. If short-circuiting occurs, there may be regions of the media where odor species may not be sufficiently metabolized by the microorganisms in the media. This may allow regions to develop where higher olfactometry and odorant concentration may be observed compared to other parts of the odor bed. This is supported by the sampling approach. At each sampling round at each location, a randomized location was chosen to give the best representation of overall odor emissions. Other contributing factors included influent composition (ratio of stormwater, high industrial load, etc.), weather (humidity and air pressure), operating conditions (including natural flux of the microbial population, fan speeds, exact stage in the treatment process and maintenance schedule/cycle) and ambient temperature.

Table 9. Validated monthly rainfall approximately $2800 \mathrm{~m}$ west of the center of the plant.

\begin{tabular}{cc}
\hline Month & Rainfall in mm (10-Year Average ${ }^{\mathbf{1}}$ ) \\
\hline October 2018 & $68.5(105)$ \\
November 2018 & $85.5(71)$ \\
December 2018 & $255(84)$ \\
January 2019 & $40(71)$ \\
February 2019 & $24(68)$ \\
March 2019 & $34(61)$ \\
April 2019 & $74.5(78)$ \\
\hline 1 10-year averaged data for this site was sourced from www metservice.com.
\end{tabular}

There are limitations to the detection of odors using olfactometry. As reported in Tables 4-7, the odor detection threshold varies greatly between different chemical species. In addition, different odorants can react to the same odorant receptors with differing affinities, causing different responses at different ratios and concentrations within mixes of odorants [28]. In addition, there is variation between individuals regarding their response to the pleasantness of an odor [27]. If an individual finds an odor offensive, their personal odor detection threshold may be lower. There may be a different olfactory response at lower, diluted concentrations then would occur if smelling the undiluted, field sample. The complexities of odor detection and individual variation may also contribute to the variation seen in this study.

A study of hydrogen sulfide retention in Nalophan bags [36] suggested that Nalophan itself not only allowed the release of hydrogen sulfide over a 30-hr retention period, the Nalophan may have also absorbed some of the hydrogen sulfide. This was demonstrated by inserting additional Nalophan material into the bag to increase the surface area [36]. The bags used for olfactometry were long sausage shaped bags that contained between 30 to $40 \mathrm{~L}$ of gas. The Nalophan bags used for SIFT-MS analysis were made from the same roll of Nalophan and held around $5 \mathrm{~L}$. Zip ties were used on either end to secure the bag shut and attach the Swagelok fittings. The gas in the SIFT-MS bags would have more surface area in contact with the Nalophan when compared to the olfactometry samples. Additionally, the samples for SIFT-MS analysis were shipped via courier to Hamilton and were not held 
in a temperature-controlled laboratory as the olfactometry samples were. Differing partial pressure within the bags due to varying rates of inflation may contribute to differing amounts of gas loss or gas mixture changes in composition. Another factor may be the formation of a gradient within the gas composition in the bag. Heavier gases such as hydrogen sulfide, may settle on the bottom of the gradient and more volatile compounds may rise to the top. This may affect the observed olfactometry and SIFT-MS results if mixing did not occur. Therefore, these differences may have resulted in a variance between the final gas composition for samples analyzed for olfactometry and SIFT-MS.

Below we discuss the applicability of both SIFT-MS and olfactometry in the different stages of the wastewater treatment process and look at ways the technologies can be used to assess odors within a plant. Individual sample IDs were referenced in this section, for which detailed data was provided in the Supplementary Materials submitted with this article.

\subsection{Influent and Primary Treatment}

Reduced sulfur compounds were expected to dominate the odor profile in the primary treatment phase [1]. Figure 6 shows a high ratio of hydrogen sulfide and methyl mercaptan in these primary treatment samples.

Screens for the primary tanks (IP-EF). Samples were acquired after biofiltration. Therefore, low concentrations of odorants were expected and were observed for most samples. However, sample IP-EF-7 displayed odor activities of 9350 and 1770 for hydrogen sulfide and methyl mercaptan, respectively. This outlier may be attributed to short-circuiting in the biofilter, where a path has developed through which air can migrate without sufficient interaction with odor-processing microbes. A section of the odor bed may have been dry and/or may require maintenance.

There was some agreement of SIFT-MS data with olfactometry results from the primary effluent samples. For the outlier, IP-EF-7, there is agreement of high OAV and strong sewage odor as characterized by the olfactory panel. Very low odor activity is observed for IP-EF-4, which is described as "indiscernible" by the panel. The SIFT-MS analysis did not detect chemical species described as displaying "vegetation" or "chemical" notes as described by the olfactory panel. The OAV data from SIFT-MS analysis for hydrogen sulfide and methyl mercaptan suggested that sewage odor compounds were the key odorants in many of the samples displaying characters described as "vegetation" or "chemical." Concentrations of these compounds were recorded at lower levels compared to IP-EF-7. However, there is a possibility that the compounds responsible for the "vegetation" and "chemical" characteristics were not analyzed for by the SIFT-MS suite (see Table 4 for a list of included compounds). The characterization notes may be attributed to the bark on the top of the biofilter. Additional analysis of volatile organic compounds emitted from the bark, such as alpha- and beta-pinene, may give insight into the contribution of the media to the odor characterization when the sewage-related compounds have been metabolized below their ODT.

Screens building (IP-SB). The two ambient samples analyzed were taken at the door of the screens building using a grab approach at nose height. OAVs of reduced sulfur compounds were low for an influent odor source consistent between the two sampling episodes. This may be attributed to the samples being ambient gas and not directly from an odor source. The "very light sewage/vegetation" attribute for IP-SB-2 correlates with the SIFT-MS analysis. Whereas the "indiscernible" character attributed to IP-SB-1 by the panel does not. A sewage odor signature was predicted from the SIFT-MS analysis to be the dominant odor character of this sample. As this was an ambient sample, odors from additional compounds to those analyzed by SIFT-MS may have been present. Ambient samples may also be more influenced by wind speed and direction and other meteorological parameters than samples taken from biofilters and liquid interfaces. Interfering odors from other parts of the site may be present that were not detected by the chosen SIFT-MS suite.

Pumping station (P-PS). Only two samples were acquired from these sources and they follow biofiltration. P-PS-1 has no hydrogen sulfide detected above instrument LOQ or odor threshold, 
while methyl mercaptan is detected in both. The "indiscernible" and "very light vegetation" characters provided by the sensory panel for samples P-PS-1 and P-PS-2 respectively, were not expected from the SIFT-MS analysis profiles. The SIFT-MS data suggested a "sewage" or "rotting vegetation" character may seem more likely (some "vegetation" character may be imparted by low levels of $\mathrm{C}_{5}$ and $\mathrm{C}_{6}$ aldehydes, however, these are lower in odor activity than the sewage contributors). Organic compounds relating to the biofilter medium may have dominated the odor character and may not have been analyzed by the wastewater-focused SIFT-MS suite used here.

Splitter box for reactor-clarifiers (P-SP). The dominate odor-active volatiles in these post-biofilter samples are again hydrogen sulfide and methyl mercaptan, with smaller contributions from a variety of $\mathrm{C}_{4}-\mathrm{C}_{6}$ aldehydes. Monoterpenes and toluene occur above the odor threshold for P-SP-4 and P-SP-3, respectively. The aldehydes detected may contribute to the "(very) light vegetation" notes frequently attributed by the sensory panel. However, the absence of "sewage" characters described by the odor panel for most of these samples was unexpected. The OAVs of both hydrogen sulfide and methyl mercaptan were expected to contribute to a sewage character. For P-SP-6, in particular, a "moderate sewage" odor character might have been expected based on the SIFT-MS results and calculated OAV. Again, organic compounds may have been present that were not analyzed via SIFT-MS. These may have contributed to the experienced odor characters in the laboratory.

\subsection{Secondary Water Treatment: Reactors/Clarifiers}

All samples from this phase were acquired using flotation sampling on the exposed liquid surface; that is, there was no biofilter to remove odorants prior to sampling. Hence, the odor measured was consistently higher in the first aerobic location (SW-A1) of the reactor/clarifier when compared to other locations further along the treatment process. SIFT-MS results reported a dominance of reduced sulfur compounds and a higher OAV in the first aerobic zone when comparing results to the aerobic zone later in the treatment process. The reduction in odor is expected as the nutrients are reduced. Little has been documented regarding the changes to odor concentration and odorant composition throughout the reactor/clarifier process. Most papers currently describe odorants from the WWTP in its entirety (e.g., [1], [2] and [37]) or with a focus on biosolids (e.g., [2]). In the second location of aerobic processing and in the final stage of anoxic processing, the total odor activity was reduced and approximately $40 \%$ of the odor measured using SIFT-MS was attributed to non-sulfur species. The reduction in measured concentrations of the volatile sulfur compounds (Table 6) concurs with this. This may indicate that most of the organic matter is removed in the first stages of the process releasing large amounts of sulfur species [1,38]. However, the reactor is zoned in a step feed process where influent is added in varying amounts to each of the anoxic zones. The first anoxic zone does contain the highest amount of primary treated influent when compared to anoxic zones 5 and 7 later in the process. To better understand the gases produced, a SIFT-MS study in conjunction with metagenomic and/or proteomic analysis of the biomass and detailed chemical analysis of the influent may be useful in determining which microbial species are producing the gases present from what substrates.

Reactor aerobic-location 1 (SW-A1). Hydrogen sulfide and methyl mercaptan dominate the odor activity of this treatment. The SIFT-MS data agree well with the odor panel, except for samples SW-A1-1 and SW-A1-4. For these samples, both hydrogen sulfide and methyl mercaptan OAVs were recorded in the 100s range, indicating a moderate to strong sewage odor would be expected. Whereas the panel attributed a "moderate earth/vegetation" note to SW-A1-1, and a "light vegetation" note to SW-A1-4. The absence of sewage notes in the descriptors provided by the sensory panel may be due to the presence of additional organic odorants.

Reactor aerobic-location 2 (SW-A2). As the wastewater at location 2 has undergone further processing compared to the wastewater at location 1 , one would generally expect lower concentrations of odor and odorant species. This was indeed the case for SW-A2-2 to SW-A2-9. Of the compounds measured using SIFT-MS, methyl mercaptan was the dominant odorant in this location. SW-A2-1 was an outlier, with high OAVs for both hydrogen sulfide and methyl mercaptan (3260 
and $1380 \mathrm{ppbV}$, respectively). Samples are collected at random and are not collected with the daily peak loads in mind. During the course of a day, loads on the reactors can differ. As the samples were collected over the course of four months, the study is designed to capture a range of seasonal and daily variation. In zone 8, increased airflow and higher dissolved oxygen set points are used to remove any remaining ammonia. Higher dissolved oxygen in this location (relative to zone 2) may be increasing the activity of a subset of the aerobic bacteria capable of generating methyl mercaptan from waste [39]. Operational processes were not monitored or included for the purpose of this study. Therefore, there is no recorded information to compare the sampling data to operational decisions. There may also be variation in the composition of the raw effluent or the microbial communities present in the reactor/clarifier sampled over the course of the sampling period.

SIFT-MS data did not support the most frequent "vegetation" character described by the panel. The SIFT-MS analysis predicted that all samples should still have a light to moderate "sewage" note. The "earth" character was not reliably predicted using SIFT-MS in this study. Additional organic species may be present that created this odor character. More research regarding the understanding of the olfactory response (from odor receptors to olfactory bulb mapping of an odor) may be required to assist in the generation of a more refined odor prediction method.

Reactor anoxic-location 1 (SW-AO). OAVs were low for the late-stage anoxic zone investigated in this study as would be expected for samples taken from the end of this treatment process where primary treated influent is added at a reduced rate than anoxic zone 1. Methyl mercaptan remained the most prevalent odorant contributor in many samples. However, methyl mercaptan was below the instrumental LOQ in several samples. It potentially may have been still above the human ODT. Other significant odorants detected were dimethyl trisulfide and $C_{2}-C_{6}$ aldehydes. However, overall chemical composition was highly variable from sample to sample in this location. Smaller aldehydes $\left(C_{1}-C_{3}\right)$ may have contributed to a chemical odor characteristic [4], in agreement with the sensory data. However, Gostelow et al. [1] describe butanal as having a "rancid/sweaty" note. For SW-AO-1 and -2 these small aldehydes have OAVs similar to those of methyl mercaptan. A better understanding of the chemical sensory signal in the olfactory system is required before we can make clearer assumptions regarding the perception of these odorants in a complex gas mix.

Clarifier (SW-CL). In general, the odor-active volatiles from this phase were similar to those of the anoxic location (SW-AO) above. This is expected as the clarifier is an anoxic environment with influent from reactor zone 8 with the purpose of settling out solids (including biomass). There was one outlier in the 20 samples analyzed (SW-CL-1). In this sample, hydrogen sulfide and methyl mercaptan OAVs were at 23 and 580, respectively. This may be due to many variable factors operationally or the chemical composition of the primary effluent. However, in spite of the presence of hydrogen sulfide and methyl mercaptan, the sensory analysis does not seem to detect the complete odor mix as a "sewage" odor. The character was described as "moderate earth". For the other samples, the comments for SW-AO apply.

\subsection{Secondary Solids Treatment}

Total OAVs were lower throughout these processes. The highest values obtained were from samples taken from the DAF. Overall the reduced sulfur compounds were significantly lower in secondary solids treatment than in primary and secondary water treatment.

Ammonia was measured marginally above the human odor threshold in a very small number of samples. Trimethylamine was detected occasionally at low ppbV throughout the treatment process. This chemical was most prevalent from biosolids stabilization onwards (that is, detected in samples taken from the biosolids storage biofilters, and the sludge stacks). Only in one of the sludge stack samples was a fishy note detected by the odor panel. The SIFT-MS odor data agrees well, with chemical odorants generating a fishy character being reported as a small fraction of the odorous chemical species analyzed. 
Vegetation characters were consistently described as the dominant odor character in the sensory analysis. SIFT-MS detected several aldehydes in these samples, although only marginally above the currently determined odor threshold. Due to high olfactory system genetic variability between people, each olfactory panel may essentially have their own unique odor threshold for different chemicals where their associated receptors may have high genetic variation within the general population. Panel members are screened for suitable odorant sensitivity using one chemical species only ( $n$-butanol). There may be other chemical species that some of the participants may be very more sensitive to due to genetic variation in their olfactory receptors. There may also be a compounding effects of multiple odorous species in the mixture on a single receptor.

Gravity thickeners (SS-GT). OAVs were low in samples taken from the biofilters in this phase. However, most samples reported measurable hydrogen sulfide and methyl mercaptan. For some samples, low molecular weight aldehydes have small, measurable odor activities. Overall a light "sewage" note would be expected, but sensory analysis reported "vegetation" or "indiscernible". SIFT-MS agrees with the latter for SS-GT-4, but not for the other samples. As discussed previously, some characters may be influenced by emissions from the biofilter materials and not analyzed by SIFT-MS in this study. These compounds may also be below the limit of detection. A study of baseline biofilter odor characteristics may assist in understanding the character described by the panel.

Dissolved air flotation (SS-AF). This was the only phase in secondary solids processing where flotation sampling was carried out. For all but SS-AF-5 (with methyl mercaptan having an OAV of 2210), odor activities were low considering that there was no biofiltration. Hydrogen sulfide was always less than an OAV of 2 in these samples, while methyl mercaptan was only detected in the above-mentioned sample, plus SS-AF-1, and SS-AF-2.

SIFT-MS analysis of sample SS-AF-2 ("light sewage") agrees well with sensory analysis, while SS-AF-5 partially agrees. The "rotten vegetation" note may be attributed to methyl mercaptan in the absence of hydrogen sulfide. Further studies of known odorant mixes may be required to determine how different odorant mixes effect odorant perception.

Digester feed (SS-DF). SIFT-MS OAV profiles predicted a "light sewage" note in contrast to the "(very) light vegetation" note as characterized by the sensory panel. As described in previous sections, the odorous gas from this part of the plant was pre-treated in an odor bed containing 30\% aged bark (see Table 1 for more details). The described "light vegetation" odor may be attributed to the microbial communities in the odor bed and the emissions from the bark. There may be compounds present that were not analyzed by SIFT-MS and hence not included in the odor profile generated.

Gas turbine stack (SS-TS). Of the three stack samples taken from the turbines, only two can be usefully discussed or compared. SIFT-MS and sensory results agree well for the first two stack samples-the "chemical" note may be attributed to the presence of low molecular weight aldehydes.

The outlying sample, SS-TS-3, may have been taken under different feed gas composition and/or stack operating conditions [40], which may have resulted in incomplete combustion as indicated by the elevated aldehyde readings. Increased alcohol concentrations in biogas can cause increased aldehyde emissions from combustion. No samples were taken of the pre-combusted biogas to provide any more insight [41].

Formaldehyde formed through combustion precludes reliable analysis of methyl mercaptan in the moist stack conditions. This is due to the water adduct of protonated formaldehyde being isobaric with protonated methyl mercaptan. In samples with high aldehyde content, only hydrogen sulfide can be measured meaningfully using SIFT-MS. Other reduced sulfur compounds that were likely present were not analyzed due to the interference of the aldehydes. Therefore, without reliable analysis of sulfur compound composition, a meaningful comparison to olfactometry could not be provided in this sample.

Centrifuge (SS-CE). Methyl mercaptan and some small aldehydes were present in the odor activity profile generated by the SIFT-MS data for the odor bed treated centrifuge plant gas. The presence of small aldehydes concurs with the "chemical" notes as detected by the sensory panel for two of 
the samples. The third sample was described by the panel as containing a "vegetation" character. The aged bark in the biofilter may have contributed to this observed odor character and may have been more dominant than the organic notes that would be expected to display "sewerage" characteristics.

Biosolids storage (SS-BS). Odor activity was low in these samples. Measured odorants were highly variable from sample-to-sample. This may be attributed to the ambient samples being collected near the door of the biosolids storage building. The wind speed and direction may contribute to the type of odorants collected as well as the disturbance of the biosolids products at the time of sampling due to mechanical movement of the biosolid material (i.e., loading lime-treated biosolid material into trucks). Much of the odorant gas may be removed by the ventilation system within the biosolids shed. The odorant gas is blown out the stacks at the rear of the building. Samples collected within the building at times of high mechanical disturbance of the biosolids may have produced a much higher result.

Dominant volatiles on occasion include hydrogen sulfide, methyl mercaptan, trimethylamine, and the low molecular weight aldehydes. Trimethylamine was expected to be present in biosolid gas due to biosolids stabilization with lime [6].

"Vegetation" and "earth" were described as the odor characters present from the olfactometry panel. "Vegetation" notes may be attributable partly to the presence of aldehydes. From the SIFT-MS analysis, a "light sewage" character would be expected due to the sulfur and rancid/putrid compounds present. In addition, the ammonia scrubber odors pass through a biofilter prior to collection. The biofilter bed was comprised of $26 \%$ Compost / Pumice. This compost component may have contributed to the "vegetation" and "earth" odor notes described by the olfactory panel.

Ammonia scrubber (SS-AS). From the ammonia scrubbers, only one sample reported levels of ammonia above the detection threshold. However, hydrogen sulfide and methyl mercaptan were also detected along with some of the smaller aldehydes. "Earth" and "vegetation" odor notes were described by the olfactometry panel. The SIFT-MS data analysis expected to see a "sewage" note as being more dominant. The scoria and compost/pumice-based odor bed may have contributed to the earthy odor characters described by the olfactometry panel.

Sludge stack (SS-SS). SIFT-MS data obtained from four samples suggested variability of the absolute and relative abundances of hydrogen sulfide and methyl mercaptan. In addition, one sample reported the highest trimethylamine measured in this study (SS-SS-2). Other reduced sulfur compounds and aldehydes were present around or slightly above the ODT.

Variance was also seen when olfactometry samples collected at different times were compared to each other. A "sewage" note was identified for SS-SS-2 and SS-SS-4. From the odor activities reported using SIFT-MS, it was predicted that the intensity for SS-SS-2 would be described as moderate as per the description for SS-SS-4. This is another example outlining the subjective nature of the olfactometry method and the complexity of odor analysis. It is difficult to assign one single odor character and choose a level of intensity that conforms to a repeatable standard. Odor fatigue can occur when large sets of samples are presented to the odor panel. The intensity descriptions may be affected by the strength and composition of the preceding samples as odors are described and characterized as a form of reference only. Only one character can be chosen by the panel.

A "light rotten fish/chemical" character was expected from the SIFT-MS results of sample SS-SS-2, as it reported elevated trimethylamine. A "light rotten fish/chemical" odor was characterized by the odor panel in sample SS-SS-3. The SIFT-MS analysis reported hydrogen sulfide levels only slightly above the ODT and several aldehydes at the ODT (which could give the "chemical note"). SIFT-MS did not report odorants associated with "chemical" or "vegetation" characters in the SS-SS-1 sample as per the olfactometry characters described. In this sample, hydrogen sulfide and methyl mercaptan were reported well above their ODTs (OAVs of 30 and $5.0 \mathrm{ppbV}$, respectively).

There are many variables that may have affected the gas composition both in the olfactometry lab and the samples provided for SIFT-MS. Samples for SIFT-MS were sent via courier to Hamilton. This drive was not temperature controlled like the laboratory conditions for the olfactometry samples. 
The resulting gas composition in the SIFT-MS samples may have changed during this trip. In addition, the surface area of Nalophan to gas sample was increased with the smaller SIFT-MS samples. As per the experiments of Eusebio et al. [36], the increased surface area may have contributed to the adsorption of hydrogen sulfide and other gases when compared to the olfactometry sample gas composition. The increase in gas volume of the samples used for olfactometry, may have assisted in keeping the chemical composition more stable. A SIFT-MS analysis of the same samples in the same location as the olfactometry panel would be more ideal when comparing both methods to reduce these variables.

There may have been compounds not analyzed via SIFT-MS that were contributing to the odor profiles and characters described by the panel. Increasing the odorous gases present in the SIFT-MS library and optimizing the analysis to include as many of these compounds as possible would be the best way to capture more compounds and determine their effect on the overall described odor.

\subsection{General Comments on Odor Analysis Using SIFT-MS}

There are limitations to this comparison as the chemical species related to earthy odor notes that feature in many low odor concentration samples are not detected using SIFT-MS. In addition, olfactometry gives you a threshold limit of detection of an odor and so complex samples with compounds that have lower ODT's can produce higher olfactometry results compared to the associated OAVs [5].

As demonstrated above, the low LOQs and selectivity of SIFT-MS make it a generally applicable tool for odor analysis at WWTPs, but it is not a single solution.

SIFT-MS can be utilized to target a wide range of odorants, as shown in Table 4, but the complex wastewater matrix does create some issues. It reliably quantifies the most notable wastewater odorants that give the "sulfur" or "sewage" odor-hydrogen sulfide and methyl mercaptan. This study demonstrated that the use of hydrogen sulfide in isolation as an indicator of odor, is not reliable. The levels of hydrogen sulfide and the more pungent sulfur odorant, methyl mercaptan, are variable amongst sources (Figure 8). Additionally, SIFT-MS can be utilized to target compounds that contribute to the "natural/fresh" or "vegetation" note, the "solvent" or "chemical" note, and the "fishy/ammonia" or "rotten fish" note. However, for these the dominant note predicted from the odor wheels (Figure 6) does not always compare well with those given informally by the sensory panel. Since most of the relevant odorants are detected by SIFT-MS at levels below the published ODTs, SIFT-MS is generally considered more reliable. However, one pungent WWTP odorant with a "chemical" note is not detected selectively: 2,3-butanedione.

For the "earthy/musty" note, SIFT-MS currently does not have sufficient selectivity to detect the extremely pungent odorants geosmin and 2-methylisoborneol of this odor character [2]. The sensory panel did not use "rancid/putrid" and "fecal/manure" descriptors at all, probably due to the common language usage that was informally employed, despite them featuring in published odor wheels $[2,4,37]$. Due to the complex matrix, SIFT-MS does not analyze several pungent compounds in these odor notes with sufficient selectivity; in particular, pentanoic acid, indole and skatole. For these compounds, plus geosmin and 2-methylisoborneol, the low ODTs compared to more generic odorants found in wastewater means that they are very susceptible to over-reporting when converted to OAVs.

Can SIFT-MS identify process abnormalities? On a sample-by-sample basis, SIFT-MS appears helpful for rapidly and reliably detecting many of the gases formed during biological processes on site. Monitoring changes in the gas composition could assist operational staff better understand changes to the biological and chemical reactions taking place in the reactors. Examples shown here include incomplete combustion in a gas turbine stack (SS-TS-3), short-circuiting in bio- or earth filters (IP-EF-7) where hydrogen sulfide is not an appropriate indicator, and elevated odor concentrations being emitted from a later stage aerobic location in a reactor-clarifier (SW-A2-1). Direct analysis on site using a mobile SIFT-MS instrument and a biofilter hood may assist in pinpointing of short circuits in bio- and earth filters, for example, and identify areas where maintenance of odor beds is required especially where hydrogen sulfide is not an appropriate indicator. 
Can SIFT-MS differentiate odor sources based on chemical analysis at the plant boundary? The large variations observed in this study for the more odorous process phases suggest that it would be very difficult to identify problems in individual processes. Preliminary evaluation of the data using multivariate statistical methods suggest that it is not feasible. However, continuous monitoring using SIFT-MS would provide immediate, sensitive indication that there is an emerging issue at the WWTP and provide a more comprehensive "odor print" than just hydrogen sulfide. In addition, the species determined by SIFT-MS in conjunction with metagenomic analysis can give insight into the microbial population within the reactors and more specific metabolic information surrounding the process. Changes to influent composition could be detected by SIFT-MS odor analysis such as a rise in odors associated with decomposing fats or animal tissues or biologically detrimental chemicals that could be toxic to activated sludge biomass.

Can SIFT-MS be used to predict the odor concentrations determined using dilution

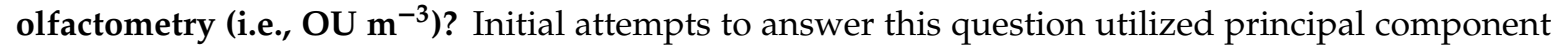
regression [42] because this is a multivariate calibration problem: i.e., prediction of odor unit based on the odorants detected by SIFT-MS. Prediction is poor no matter what approach is taken (e.g., using individual odorant OAVs or grouping those odorants of similar odor note). There are likely two reasons for this. First, SIFT-MS is not analyzing all odorants effectively, with the absence of reliable "earthy/musty" note likely being the most significant. A sample of "moderate earth" character (SS-AF-3) illustrates this situation, with an odor concentration of 11,205 $\mathrm{OU} \mathrm{m}^{-3}$ but a total odor activity of only $4 \mathrm{ppbV}$ calculated from the dominant odorants analyzed with SIFT-MS. Instead a simple correlation of total odor activity value for the dominant, reliably analyzed odorants with odor concentration was attempted (Figure 7). There is a trend indicating general agreement between olfactometry and the total odor activity, but since SIFT-MS is not quantifying compounds reliably that have "earthy/musty" notes, there are also significant outliers, such as the one mentioned above.

Braithwaite [5] questions the reliability of dynamic dilution olfactometry, despite it forming the basis of numerous standard methods globally (e.g., AS/NZS 4323.3:2001, BS EN 13725). Braithwaite [5] summarizes the situation:

"As an odor mixture is diluted, the odor notes and hedonic tones change and eventually, after sufficient dilution, the concentration of the final detectable odorant drops below its odor detection threshold, so the diluted sample becomes 'odorless'. The amount of dilution required to reach this point is considered an indicator of the odor intensity of the initial, undiluted sample, which is problematic because the final detectable odorant may not be indicative of the odorant that dominated the odor of the undiluted sample (nor the dominant odorant in the partially diluted sample at the fenceline). Relying on dilution quantities to indicate the intensity of the total odor is crude at best and misleading at worst."

Can SIFT-MS predict the odor note? The results presented here show agreement for less than half of the samples tested. Agreement is primarily in terms of the "sewage", "chemical" and "vegetation" notes provided informally by the sensory panel. However, the absence of "sewage" notes from the sensory panel in many samples where SIFT-MS detects hydrogen sulfide and methyl mercaptan as dominant odor-active volatiles suggests that the informal approach to odor note determination is a crude description of the odor. The SIFT-MS analytical suites were chosen based upon expected wastewater odorants and may not have analysed every odorous compound associated with bark and soil in the biofilters. Hence no firm conclusions can be made at present. Another point to consider is the change in odors present in the sample from time of sampling to analysis. While samples are analyzed within the 30-h window, their chemical composition can likely be altered over this period as different gases interact with each other and other gases are absorbed into the Nalophan. A field SIFT-MS instrument would eliminate this issue.

Can SIFT-MS predict odor intensity and hedonic tone? Odor intensities listed in Tables 5-7 and the supplementary data are from informal sensory measurements based upon standard odor detection threshold methods. The panel did not evaluate hedonic tones. The data, therefore, do not facilitate 
evaluation of intensity and hedonic tone versus SIFT-MS measurements. This will need to be evaluated in another study.

\section{Conclusions}

The wastewater matrix is challenging due to its complexity and variability. SIFT-MS selectively detects and quantifies many, but not all, of the key odorants. The SIFT-MS data correlate well with the literature reviewing the important odorants in WWTP process, except for the "earthy/musty" odor, which cannot be selectively analyzed currently. Characteristic "sulfur" (or "sewage") odor is readily and sensitively detected, even in samples where informal sensory analysis misses it. SIFT-MS unequivocally demonstrates that hydrogen sulfide is not a reliable sole marker for WWTP odor.

Total OAVs determined using SIFT-MS correlate loosely with the odor concentration determined by sensory panel using dynamic dilution olfactometry. Samples that do not correlate well are attributed to the inability of SIFT-MS to detect "earthy/musty" notes effectively in the WWTP matrix and to the known limitations with this sensory method.

Compared with other analytical techniques used for odor analysis, the results obtained with SIFT-MS compatible with the number of compounds that can be detected at below or approaching human ODTs. However, SIFT-MS does not provide a comprehensive solution for WWTP odor analysis. Nevertheless, because fenceline odors are more frequently attributed to the "sulfur" or "sewage" note, than any other note, there are applications for SIFT-MS in WWTPs. These include continuous monitoring at the fenceline to provide rapid indication of developing odor issues so that they can be resolved before they become a major issue with neighbors, and the possibility of mobile instrument operation on-site to identify the source of an odor issue.

Braithwaite [5] has recently recommended SIFT-MS as part of a multi-methodology approach for odor research, in which SIFT-MS is combined with GC-Sensory (GC-MS/O) and the odor profile method (OPM) to provide the most comprehensive research portfolio available. As Brathwaite points out, OPM is the leading sensory technique, and GC-MS/O is the leading combined instrumental technique. However, both OPM and GC-MS/O are slow, off-line, and expensive per sample. SIFT-MS complements them by providing real-time, targeted analysis of most odorants to near or below the ODT, making continuous monitoring of odor possible in the field, so that even transient events are detected.

Supplementary Materials: The following are available online at http://www.mdpi.com/2076-3298/7/10/90/s1: Supplementary Data-SENSORY and SIFT-MS OAV Data.xlsx; Supplementary Data-Table 4 references.pdf.

Author Contributions: This study was conceptualized by V.S.L and C.B. The SIFT-MS methodology was developed by V.S.L. and the sensory methodology by C.B. Data analysis was conducted by V.S.L. V.S.L. drafted the initial paper and C.B. and M.J.M. reviewed and substantially edited it. All authors have read and agreed to the published version of the manuscript.

Funding: This research received no external funding.

Acknowledgments: We thank David Steiner and Liam Templeton of Watercare Laboratory Service/Watercare Services Limited for developing the site sampling plan and collecting the odor samples. Anatoly Chernyshev and Nick Kuan of Analytica Laboratories (Hamilton, New Zealand) conducted the SIFT-MS analyses and Nina Hardy and the sensory panel at the Watercare Laboratory Services (Auckland, New Zealand) for conducting the odor analyses. The authors are grateful to Diandree Padayachee (Syft Technologies) for helpful discussions.

Conflicts of Interest: VL is an employee of, and MM a consultant to, Syft Technologies Limited, a manufacturer of commercial SIFT-MS instruments. CB declares no conflict of interest.

\section{References}

1. Gostelow, P.; Parsons, S.A.; Stuetz, R.M. Odour measurements for sewage treatment works. Wat. Res. 2001, 35, 579-597. [CrossRef]

2. Fisher, R.M.; Barczak, R.J.; Suffet, I.H.; Hayes, J.E.; Stuetz, R.M. Framework for the use of odour wheels to manage odours throughout wastewater biosolids processing. Sci. Total Environ. 2018, 634, $214-223$. [CrossRef] [PubMed] 
3. Zaouak, O.; Ben Daoud, A.; Fages, M.; Fanlo, J.; Aubert, B. High performance, cost effective miniature sensor for continuous network monitoring of $\mathrm{H}_{2}$ S. Chem. Eng. Trans. 2012, 30, 325-330.

4. Suffet, I.H.; Burlingame, G.A.; Rosenfeld, P.E.; Bruchet, A. The value of an odor-quality-wheel classification scheme for wastewater treatment plants. Wat. Sci. Tech. 2004, 50, 25-32. [CrossRef]

5. Braithwaite, S.K. Sensory Analysis and Health Risk Assessment of Environmental Odors. Ph.D. Thesis, University of California-Los Angeles, Los Angeles, CA, USA, 2019.

6. Fisher, R.M.; Alvarez-Gaitan, J.P.; Stuetz, R.M. Review of the effects of wastewater biosolids stabilization processes on odor emissions. Crit. Rev. Environ. Sci. Tech. 2019, 49, 1515-1586. [CrossRef]

7. Mannebeck, D. Olfactometers according to EN 13725. In Springer Handbook of Odor; Buettner, A., Ed.; Springer: Cham, Switzerland, 2017; pp. 545-551.

8. McEwan, M.J. Direct analysis mass spectrometry. In Ion Molecule Attachment Reactions: Mass Spectrometry; Fujii, T., Ed.; Springer: New York, NY, USA, 2015; pp. 263-317.

9. Biasioli, F.; Gasperi, F.; Yeretzian, C.; Märk, T.D. PTR-MS monitoring of VOCs and BVOCs in food science and technology. Trends Anal. Chem. 2011, 30, 968-977. [CrossRef]

10. Biasioli, F.; Gasperi, F.; Odorizzi, G.; Aprea, E.; Mott, D.; Marini, F.; Autiero, G.; Rotondo, G.; Mark, T.D. PTR-MS monitoring of odour emissions from composting plants. Int. J. Mass Spectrom. 2004, 239, 103-109. [CrossRef]

11. Biasioli, F.; Aprea, E.; Gasperi, F.; Mark, T.D. Measuring odour emission and biofilter efficiency in composting plants by proton transfer reaction-mass spectrometry. Wat. Sci. Tech. 2009, 59, 1263-1269. [CrossRef]

12. Cappellin, L.; Loreto, F.; Aprea, E.; Romano, A.; Sanchez del Pulgar, J.; Gasperi, F.; Biasioli, F. PTR-MS in Italy: A multipurpose sensor with applications in environmental, agri-food and health science. Sensors 2013, 13, 11923-11955. [CrossRef]

13. Feilberg, A.; Bildsoe, P.; Nyord, T. Application of PTR-MS for measuring odorant emissions from soil application of manure slurry. Sensors 2015, 15, 1148-1167. [CrossRef]

14. Liu, D.; Nyord, T.; Rong, L.; Feilberg, A. Real-time quantification of emissions of volatile organic compounds from land spreading of pig slurry measured by PTR-MS and wind tunnels. Sci. Total Environ. 2018, 639, 1079-1087. [CrossRef] [PubMed]

15. Hansen, M.J.; Adamsen, A.P.S.; Pedersen, P.; Feilberg, A. Prediction of odor from pig production based on chemical odorants. J. Environ. Qual. 2012, 41, 436-443. [CrossRef]

16. Liu, D.; Feilberg, A.; Nielsenn, A.M.; Adamsen, A.P.S. PTR-MS measurement of partition coefficients of reduced volatile sulfur compounds in liquids from biotrickling filters. Chemosphere 2013, 90, 1396-1403. [CrossRef] [PubMed]

17. Prince, B.J.; Milligan, D.B.; McEwan, M.J. Application of selected ion flow tube mass spectrometry to real-time atmospheric monitoring. Rapid Commun. Mass Spectrom. 2010, 24, 1763-1769. [CrossRef]

18. Hera, D.; Langford, V.S.; McEwan, M.J.; McKellar, T.I.; Milligan, D.B. Negative reagent ions for real time detection using SIFT-MS. Environments 2017, 4, 16. [CrossRef]

19. Langford, V.S.; McEwan, M.J.; Askey, M.; Barnes, H.A.; Olerenshaw, J.G. Comprehensive instrumental odor analysis using SIFT-MS: A case study. Environments 2018, 5, 43. [CrossRef]

20. Spanel, P.; Smith, D. Selected ion flow tube: A technique for quantitative trace gas analysis of air and breath. Med. Biol. Eng. Comput. 1996, 24, 409-419. [CrossRef]

21. Smith, D.; Spanel, P. Selected ion flow tube-mass spectrometry (SIFT-MS) for on-line trace gas analysis. Mass Spec. Rev. 2005, 24, 661-700. [CrossRef]

22. Langford, V.S.; Graves, I.; McEwan, M.J. Rapid monitoring of volatile organic compounds: A comparison between gas chromatography/mass spectrometry and selected ion flow tube mass spectrometry. Rapid Commun. Mass Spectrom. 2014, 28, 10-18. [CrossRef]

23. Moon, S.Y.; Cliff, M.A.; Li-Chan, E.C.Y. Odour-active components of simulated beef flavour analysed by solid phase microextraction and gas chromatography-mass spectrometry and -olfactometry. Food Res. Int. 2006, 39, 294-308. [CrossRef]

24. Bulliner, E.A., IV; Koziel, J.A.; Cai, L.; Wright, D. Characterization of livestock odors using steel plates, solid-phase microextraction, and multidimensional gas chromatography-mass spectrometry-olfactometry. J. Air Waste Manag. 2006, 56, 1391-1403. [CrossRef] [PubMed]

25. Niimura, Y.; Nei, M. Evolution of olfactory receptor genes in the human genome. Proc. Natl. Acad. Sci. USA 2003, 100, 12235-12240. [CrossRef] [PubMed] 
26. Gilad, Y.; Bustamante, C.D.; Lancet, D.; Pääbo, S. Natural selection on the olfactory receptor gene family in humans and chimpanzees. Am. J. Hum. Genet. 2003, 73, 489-501. [CrossRef]

27. Knaapila, A.; Keskitalo, K.; Kallela, M.; Wessman, M.; Sammalisto, S.; Hiekkalinna, T.; Palotie, A.; Peltonen, L.; Tuorila, H.; Perola, M. Genetic component of identification, intensity and pleasantness of odours: A Finnish family study. Eur. J. Hum. Genet. 2007, 15, 596-602. [CrossRef]

28. Belhassan, A.; Zaki, H.; Chtita, S.; Benlyas, M.; Lakhlifi, T.; Bouachrine, M. Study of interactions between odorant molecules and the hOR1G1 olfactory receptor by molecular modeling. Egypt. J. Ear Nose Throat Allied Sci. 2017, 18, 257-265. [CrossRef]

29. Li, Y.R.; Matsunami, H. Unfolding the mystery of olfactory receptor gene expression. Develop. Cell 2003, 27, 128-129. [CrossRef]

30. Standards Australia and Standards New Zealand. Determination of Odour Concentration by Dynamic Olfactometry (AS/NZS 4323.3:2001); Standards Australia International Ltd.: Sydney, Australia; Standards New Zealand: Wellington, New Zealand, 2001.

31. Standards Australia and Standards New Zealand. Method 4: Area Source Sampling-Flux Chamber Technique (AS/NZS 4323.4:2009); Standards Australia International Ltd.: Sydney, Australia; Standards New Zealand: Wellington, New Zealand, 2009.

32. United States Environmental Protection Agency. Measurement of Gaseous Emission Rates from Land Surfaces Using an Emission-Isolation Flux Chamber; User's guide, EPA Contract No. 68-02-3889; Radian Corporation: Nashville, TN, USA, February 1986.

33. van Gemert, L.J. Odour Thresholds: Compilations of Odour Threshold Values in Air, Water and Other Media; Oliemans, Punter and Partners: Utrecht, The Netherlands, 2011.

34. Nagata, Y. Measurement of odor threshold by triangle odor bag method. In Odor Measurement Review; Japan Ministry of the Environment, Ed.; Government of Japan: Tokyo, Japan, 2003; pp. 118-127.

35. Vitko, T.; Dillon, C.; Cowden, S.; Erdal, Z.; Witherspoon, J.; Zhou, Y.; Suffet, I.H. Innovative odor control master planning sets the stage for targeted foul air treatment. Water Environ. Technol. 2017, 29, $42-47$.

36. Eusebio, L.; Capelli, L.; Sironi, S. $\mathrm{H}_{2} \mathrm{~S}$ Loss through Nalophan ${ }^{\mathrm{TM}}$ bags: Contributions of adsorption and diffusion. Sci. World J. 2017, 2017, 9690704. [CrossRef]

37. Suffet, I.H.; Rosenfeld, P. The anatomy of odour wheels for odours of drinking water, wastewater, compost and the urban environment. Wat. Sci. Tech. 2007, 55, 335-344. [CrossRef]

38. Sun, S.; Jia, T.; Chen, K.; Peng, Y.; Zhang, L. Simultaneous removal of hydrogen sulfide and volatile organic sulfur compounds in off-gas mixture from a wastewater treatment plant using a two-stage bio-trickling filter system. Front. Environ. Sci. Eng. 2019, 13, 60. [CrossRef]

39. Herter, C.A. The production of methyl mercaptan by fecal bacteria grown on a peptone medium. J. Biol. Chem. 1906, 1, 421-424.

40. Ghenai, C.; Janajreh, I. Combustion of renewable biogas fuels. J. Energy Power Eng. 2015, 9, 831-843.

41. Agarwal, A.K. Biofuels (alcohols and biodiesel) applications as fuels for internal combustion engines. Prog. Energy Combust. Sci. 2007, 33, 233-271. [CrossRef]

42. Viaene, J.; Heyden, Y.V. Introduction to herbal fingerprinting by chromatography. In Chemometrics in Chromatography; Komsta, Ł., Heyden, Y.V., Sherma, J., Eds.; CRC Press: Boca Raton, FL, USA, 2018; pp. 351-370.

Publisher's Note: MDPI stays neutral with regard to jurisdictional claims in published maps and institutional affiliations.

(C) 2020 by the authors. Licensee MDPI, Basel, Switzerland. This article is an open access article distributed under the terms and conditions of the Creative Commons Attribution (CC BY) license (http://creativecommons.org/licenses/by/4.0/). 